\title{
Distinguishing interstitial and substitutional diffusion in grand-potential based phase-field model
}

\author{
P G Kubendran Amos ${ }^{\mathrm{a}, *}$, Britta Nestler ${ }^{\mathrm{a}, \mathrm{b}}$ \\ ${ }^{a}$ Institute of Applied Materials (IAM-CMS), Karlsruhe Institute of Technology (KIT), \\ Strasse am Forum 7, 76131 Karlsruhe, Germany \\ ${ }^{b}$ Institute of Digital Materials Science (IDM), Karlsruhe University of Applied Sciences, \\ Moltkestr. 30, 76133 Karlsruhe, Germany
}

\begin{abstract}
Grand-potential based phase-field technique is often claimed to be an efficient approach for modelling phase transformation in multicomponent systems. Since this technique largely employs mole fraction to treat concentration, it is principally restricted to simulating microstructural evolutions which are exclusively governed by substitutional diffusion. In this work, an existing grand-potential model is re-formulated to encompass interstitial diffusion. The distinction between interstitial and substitutional diffusion is achieved by adopting molar number-density $\left(\mathrm{mol} / \mathrm{m}^{3}\right)$ based description of composition. The ability of the re-formulated approach to model phase transformation accompanying interstitial and substitutional diffusion is elucidated by simulating rather straightforward decomposition of austenite into ferrite in the ternary Fe-C-Mn system. Energy-density approximations that facilitate the incorporation of CALPHAD data in the present framework are delineated for general, and in particular for Fe-C-X alloy systems. Furthermore, phase-change under para-equilibrium, which can only be imposed through the re-formulated variant of grand-potential technique, is modelled and the resulting concentration profile is discussed in comparison to the outcomes of the conventional approach. Partitioning of carbon in constrained-carbon-equilibrium condition is consistently simulated in-line with its description.
\end{abstract}

Keywords: Substitutional and interstitial diffusion, reconstructive phase-transformation, Fe-C-X system, para-equilibrium, Constrained-Carbon-Equilibrium, molar number density 


\section{Introduction}

In any given alloy, desired properties are achieved through appropriate processing techniques. A processing technique like heat treatment can only be devised with an adequate understanding of the microstructural evolution exhibited by the material [1]. Temporal changes in a microstructure, under a definite condition, are rarely straightforward to be exclusively analysed through experimental techniques. To that end, theoretical treatments are largely adopted to complement the experimental observation, and enhance the understanding of microstructural transformations. Particularly, with the mounting availability of affordable resources, computational techniques have increasingly been adopted to delineate complex microstructural evolution [2]. Phase-field modelling is one such numerical approach which is steadily gaining ground as a go-to technique for analysing microstructural evolutions in wide-range of materials [3].

\subsection{Sharp-interface treatment}

Any microstructural changes can be theoretically viewed as a free-boundary problem, and thus, treated analogously to the canonical Stefan's problem [4, 5]. Accordingly, for a system with well-posed boundary and initial conditions, a phase transformation is modelled by solving the temporal evolution of the fundamental variable, under corresponding free-boundary (or Stefan) and equilibrium (or Dirichlet) condition. In this approach, an interface with 'zero' thickness, characterised by an abrupt change in the fundamental variable, distinguishes the constituent phase of the microstructure. A phase transformation is thus perceived by tracking the evolution of the sharp interface. Owing to the unambiguous nature of the underlying formulation, the sharp-interface treatment is relatively well-established. However, the apparent straightforward formulation involved in this technique gets increasingly convoluted with the complexity of the evolving microstructure [6]. Therefore, phase-field approach is employed as an elegant alternate for modelling phase transformations that yield intricate microstructures.

\footnotetext{
*Prince Gideon Kubendran Amos

Email address: prince.amos@kit .edu (P G Kubendran Amos)
} 


\subsection{Phase-field modelling}

In phase-field formalism, fundamental parameter(s) describing a system and/or its state is accompanied by an additional scalar variable. This scalar variable, called phase-field $(\phi)$, assumes a constant value within a given phase, while monotonously varying across the interface. In other words, the interface is characterised by the smooth transition of the scalar variable- $\phi$, and correspondingly, diffuse interface with a definite width is associated with phase-field modelling. By introducing phase-field, the microstructural changes are no longer realised by tracking the interface but rather through the spatio-temporal evolution of the additional scalar-variable, $\phi(\boldsymbol{x}, t)$ [7, 8, 9]. Furthermore, treating phase-field as volume fraction of given phase, such that $\sum \phi(\boldsymbol{x})=1$ at any given location $\boldsymbol{x} \in V$, where $V$ is the volume of the system, a two phase microstructural evolution can be effectively modelled by a single variable.

The entire domain, in phase-field approach, is distinguished into bulk phase, wherein phasefield is spatially independent, and diffuse interface characterised by the transition of $\phi(\boldsymbol{x})$. Accordingly, the overall energy of a two-phase system is expressed in the form of Ginzburg-Landau type functional as

$$
\mathcal{F}(\phi, \nabla \phi, \boldsymbol{\eta})=\int_{\mathrm{d} V} \mathrm{~d} V\left[f_{\text {intf }}(\phi, \boldsymbol{\nabla} \phi)+f_{\text {bulk }}(\boldsymbol{\eta}, \phi)\right],
$$

where $f_{\text {intf }}(\phi, \nabla \phi)$ and $f_{\text {bulk }}(\boldsymbol{\eta}, \phi)$ represent the energy-density contribution of the diffuse-interface and bulk region, respectively [10, 11]. Moreover, in Eqn. (1), $\boldsymbol{\eta}((x), t)$ is the fundamental variable which is appropriately chosen to describe a given system and its evolution.

Having defined the overall energy-density through the functional $\mathcal{F}(\phi, \nabla \phi, \boldsymbol{\eta})$ in Eqn. (1), the system is allowed to evolve towards its progressive phenomenological decrease. Therefore, the temporal evolution of phase-field, which manifests itself as the perceived microstructural changes, is invariably expressed as the variational derivative of the energy-density functional,

$$
\frac{\partial \phi(\boldsymbol{x}, t)}{\partial t}=-\tilde{M} \frac{\delta \mathcal{F}(\phi, \boldsymbol{\nabla} \phi, \boldsymbol{\eta})}{\delta \phi},
$$

where $\tilde{M}$ dictates the mobility of phase-field. Depending on the nature of the transformation, the fundamental variable, $\boldsymbol{\eta}$, and its evolution is appropriately delineated. Moreover, for microstructural transformations wherein the volume-fraction of the phases do not temporally-vary, phasefield is treated as a conserved variable, and Eqn. (2) adopts a substantially different form [12]. 
However, since the present work primarily focuses on phase transformation, subsequent discussions adhere to Eqn. (2) describing non-conserved evolution of phase-field [13].

\subsubsection{Pure-substance approach}

Early attempts to model solidification in alloys, which are characterised by one or more independent concentrations, can be viewed as a direct extension of phase-field approach adopted for analysing pure substances [14, 15]. Phase transformation in pure substances is conventionally modelled by considering the temperature as the dynamic and continuous variable, in addition to phase-field. Adhering to this framework, a phase-field treatment for alloy solidification is formulated by treating concentration as the continuous variable [16, 17]. In a system, wherein concentration is the continuous variable, the contribution from the bulk phases reads

$$
f_{\text {bulk }}(\boldsymbol{c}, \phi)=f^{\alpha}(\boldsymbol{c}) h(\phi)+f^{\beta}(\boldsymbol{c})[1-h(\phi)]
$$

where $h(\phi)$ is a smooth and monotonously varying interpolation function. Owing to the multicomponent nature of the system, concentration is expressed as a tuple of $m$-components, $\boldsymbol{c}(\boldsymbol{x}, t)=$ $\left\{c_{i}(\boldsymbol{x}, t), c_{j}(\boldsymbol{x}, t), \ldots, c_{m}(\boldsymbol{x}, t)\right\}$, with $m$ representing total number of alloying elements including the solvent. Moreover, $\alpha$ and $\beta$ are evolving (solid) and parent phase (liquid), respectively. Despite the apparent thermodynamic consistency, the limitation of this approach gets evident when considering a system in chemical equilibrium [18].

Chemical equilibrium in a two-phase microstructure is established when the composition of the phases pertain to the characteristic equilibrium concentration. Correspondingly, an onedimensional system in equilibrium can be expressed as

$$
\begin{array}{rlrl}
\phi_{0}^{+}=1 ; & \boldsymbol{c} & =\boldsymbol{c}_{\mathrm{eq}}^{\alpha} \equiv\left\{c_{i \mathrm{eq}}^{\alpha}, c_{j \mathrm{eq}}^{\alpha}, \ldots, c_{k: \mathrm{eq}}^{\alpha}\right\} \\
\phi_{0}^{-}=0 ; & \boldsymbol{c}=\boldsymbol{c}_{\mathrm{eq}}^{\beta} \equiv\left\{c_{\text {ieq }}^{\beta}, c_{\text {jeq }}^{\beta}, \ldots, c_{k \text { :eq }}^{\beta}\right\},
\end{array}
$$

wherein the diffuse interface is characterised by $\phi_{0}(x) \in(0,1)$, and $\boldsymbol{c}\left(\phi_{0}\right)$ representing a smooth transition between the equilibrium compositions. Moreover, $c_{i \text { :eq }}^{\Theta}$ with $\Theta \in\{\alpha, \beta\}$, indicates equilibrium concentration of component- $i$ which is hugely dependent on phases. 
Under chemical equilibrium, with the system devoid of any influence of the curvature, the interface remains static $\left(\frac{\partial \phi}{\partial t}=0\right)$. Therefore, for an interface contribution of the form

$$
f_{\text {intf }}(\phi, \nabla \phi)=w_{1}|\nabla \phi|^{2}+w_{2} f_{\text {pen }}(\phi)
$$

where $f_{\text {pen }}(\phi)$ is well- or obstacle-type penalising term with $w_{1}$ and $w_{2}$ corresponding to the representative coefficients of gradient- and potential-energy densities, Eqns. (3) and (5), through Eqn. (2) yield a relation

$$
2 w_{1} \nabla^{2} \phi_{0}-w_{2} \frac{\mathrm{d} f_{\text {pen }}\left(\phi_{0}\right)}{\mathrm{d} \phi_{0}}=\frac{\mathrm{d} \Delta_{\mathrm{ch}}\left(\boldsymbol{c}\left(\phi_{0}\right), \phi_{0}\right)}{\mathrm{d} \phi_{0}} .
$$

The chemical driving-force on the right side of above expression reads

$$
\Delta_{\mathrm{ch}}\left(\boldsymbol{c}\left(\phi_{0}\right), \phi_{0}\right)=f^{\alpha}\left(\boldsymbol{c}\left(\phi_{0}\right)\right) h\left(\phi_{0}\right)+f^{\beta}\left(\boldsymbol{c}\left(\phi_{0}\right)\right)\left[1-h\left(\phi_{0}\right)\right]-\sum_{i}^{m-1} \mu_{i}^{\mathrm{eq}} c_{i}\left(\phi_{0}\right) .
$$

where $\mu_{i}^{\mathrm{eq}}$ represents the equilibrium diffusion potential of component- $i$. Here, it is important to realise that, while the chemical potential of a component $i$, with its conventional description, is represented as $\tilde{\mu}_{i}$ in the present formalism, its corresponding diffusion potential, which is defined as the difference in a chemical potential of a component to its matrix, is denoted by $\mu_{i}\left(=\tilde{\mu}_{i}-\tilde{\mu}_{\text {matrix }}\right)$.

Despite the equivalent energy-density established between the phases in a chemical equilibrium, it is evident from Eqns. (6) and (7) that a remnant driving-force persists, which augments to the interface contribution as excess energy. This limitation of the pure-substance formulation is discussed as a non-physical coupling of the interface and bulk driving-force, that relates the associated coefficients and hinders in recovering material properties [18]. Additionally, the coupling of the interface and bulk contribution poses a serious constraint on interface width, and size of the simulation domain [19].

\subsubsection{Two-phase approach}

Effective decoupling of the bulk and interface contributions can be established by ensuring that the right-hand side of Eqn. (6) vanishes in equilibrium. This has successfully been achieved by introducing 'fictitious' phase-dependent concentrations, $\boldsymbol{c}^{\Theta}=\left\{c_{i}^{\Theta}, c_{j}^{\Theta} \ldots, c_{k}^{\Theta}\right\}$, where $\Theta \in$ 
$\{\alpha, \beta\}[18,20]$. The underlying principle for introducing phase-dependent concentration is the assumption that, across the diffuse interface, the phases are distinct and are in local equilibrium, unlike the pure-substance approach, wherein the phases are mixed with a single continuous concentration [21]. By formulating the bulk contribution based on phase-dependent concentrations as

$$
f^{\alpha}\left(\boldsymbol{c}_{\mathrm{eq}}^{\alpha}\right)-\sum_{i}^{k-1} \mu_{i}^{\mathrm{eq}} c_{i \text { :eq }}^{\alpha}=f^{\beta}\left(\boldsymbol{c}_{\mathrm{eq}}^{\beta}\right)-\sum_{i}^{k-1} \mu_{i}^{\mathrm{eq}} c_{i \text { :eq }}^{\beta}
$$

the two-phase treatment ensures that the bulk driving-forces vanish in chemical equilibrium. Consequently, the interface and bulk contributions decouple, and Eqn. (4) transforms to

$$
2 w_{1} \nabla^{2} \phi_{0}=w_{2} \frac{\mathrm{d} f_{\text {pen }}\left(\phi_{0}\right)}{\mathrm{d} \phi_{0}} .
$$

Eventhough this two-phase approach eliminates any non-physical influence of bulk contribution on the interface, it significantly adds to the computational cost of the overall numerical treatment. In other words, by introducing previously unknown variables, the two-phase technique appends a supplementary step, wherein these variables are determined. The unknown phase-dependent concentrations, for every component, are ascertained from the homogeneous concentration, expressed as

$$
c_{i}=c_{i}^{\alpha} h(\phi)+c_{i}^{\beta}[1-h(\phi)] \quad \forall \quad i,
$$

through the assumed equilibrium, which introduces the relation $\mu_{i}^{\alpha}=\mu_{i}^{\beta}=\mu_{i}^{\mathrm{eq}} \quad \forall i$. Having realised the ability of the two-phase treatment in eliminating the non-physical interaction of the bulk and interface contribution, subsequent advancements focus primarily on identifying the unknown variables, $c^{\Theta}$, efficiently.

\subsubsection{Grand-potential approach}

Motivated by the undergirding principles of the pure-substance treatment of solidification, and understanding the bulk driving-forces which emerge from the two-phase approach, a seemingly efficient alternate has been discussed for modelling phase transformation in alloys. This approach re-formulates the overall energy functional, in Eqn. (1), by adopting grand-potential 
density to describe the contribution of the bulk phases [21, 22, 23]. In the grand-potential framework, instead of concentration, the diffusion (or chemical) potential of the components is treated as the fundamental variable. Given the invertibility of the free-energy density of the individual phases, the unknown phase-dependent concentrations are efficiently calculated.

\subsubsection{Existing phase-field models realising substitutional and interstitial diffusion}

In all the aforementioned techniques, which are directed towards modelling microstructural transformation in multicomponent systems, the concentration $\left(c^{\Theta}\right)$ is largely treated in mole fractions. The limitations of representing concentration in mole fraction, in the framework of phase-field models, have previously been discussed in great detail [24]. These restriction include the in-adequacy of the approach to distinguish the lattice occupancy of the alloying elements. In other words, the interstitial and substitutional diffusion of the components cannot be sufficiently distinguished when composition is treated in mole fraction. Consequently, such phase-field techniques are confined to phase transformation governed by local equilibrium condition and cannot model unique conditions, like para-equilibrium, which is characterised by the diffusion of few, and specific, alloying elements [25]. Having realised this potential limitation, different representations have been rendered for concentration. Preliminary works adopt molar number-density, $\rho^{\Theta}$, which describes concentration as number of moles per unit volume, in puresubstance approach to obviate the restriction imposed by mole fraction [24, 26]. This has subsequently been extended to the two-phase treatment [27]. Recently, a multiphase-field technique with the ability to model microstructural evolution in systems with sublattices is elegantly presented in Ref. [28]. This technique adopts site-fraction, instead of number-density, to treat concentration. Eventhough these models are thermodynamically consistent, they invariably employ two-phase treatment, and consequently, incur a computational burden of estimating the previously unknown phase-dependent concentration. Therefore, in this work, a grand-potential based phase-field approach, which expresses concentration in molar number-density, is presented to distinguish interstitial and substitutional diffusion. 


\section{Model extension}

Phase-field techniques, which hitherto adopt molar number-density to describe the chemical composition of a system, begin by comprehensively elucidating the limitations of using mole fraction, and proceed by re-formulating the approach based on the alternate parameter [24, 26]. Having assumed a prevalent understanding, such description, while convincingly delineating the need for a different treatment of concentration, seemingly overlooks a proper introduction to number-density, and its relation to the conventionally used mole fraction. Particularly, given that molar number-density is primarily described based on volume, a re-formulation involving molar volume would conceivably be more pertinent, and advantageous, owing its existing critical-role in converting the free-energy densities, and incorporating quantitative driving-force [29]. Therefore, this section begins with a rather pedagogical introduction to molar number-density, which facilitates in deriving a relation between the two concentration parameters (mole fraction and number-density) through molar volume.

\subsection{Molar number-density}

At any given instant during a transformation, volume of a phase is dictated by the number of moles of certain, or all, constituent components. Correspondingly, for a phase- $\alpha$ comprising of $m$ chemical species, its volume can be expressed as

$$
\sum_{i=1}^{m} v_{i}^{\alpha} N_{i}^{\alpha}(t)=V^{\alpha}(t)
$$

where $N_{i}^{\alpha}$ is the number of mole of alloying element $i$. The description of volume in Eqn. (11) stems from the assumption that all chemical components associated with phase- $\alpha$ contribute to $V^{\alpha}(t)$. The contribution of an individual species to volume, from Eqn. (11), can be quantified as

$$
\frac{\partial V^{\alpha}}{\partial N_{i}^{\alpha}}=v_{i}^{\alpha}
$$

where $v_{i}^{\alpha}$ is referred to as partial molar-volume of component- $i$ in phase- $\alpha$.

When a phase includes more than one sub-lattice, with the chemical-species assuming a characteristic partial molar-volume in each of these sub-lattices, Eqn. 111 is extended to describe its 
volume. Accordingly, volume of phase- $\alpha$, which encompasses $q$ sub-lattices is written as

$$
\sum_{p=1}^{q} \sum_{i=1}^{m} v_{p: i}^{\alpha} N_{p: i}^{\alpha}(t)=V^{\alpha}(t)
$$

where $v_{p: i}^{\alpha}$ is the characteristic partial molar-volume of $i$ occupying sub-lattice $p$. From Eqn. (13), volume of phase- $\alpha$ comprising of chemical species which occupy regular or interstitial sites can be appropriately formulated. However, it should be noted that, while Eqn. (13) permits the possibility of a component occupying more than one sub-lattices, analogous expression delineating volume of phase- $\alpha$ with active interstitial-sites should ensure that each chemical species is exclusively bound to one sub-lattice. In other words, the formulation to estimate volume of phase- $\alpha$ with regular and interstitial sub-lattice should corroborate the condition that a component occupying a given lattice is restricted to it, and is not associated with the other site.

Volume of phase- $\alpha$, which consist of $j$ and $l$ components respectively occupying regular and interstitial sites, is expressed as

$$
\sum_{i=1}^{j} v_{\text {sub: } i}^{\alpha} N_{\text {sub: } i}^{\alpha}+\sum_{k=1}^{l} v_{\text {ints: } k}^{\alpha} N_{\text {ints: } k}^{\alpha}=V^{\alpha} .
$$

In the above formulation, the partial molar-volume of components $i$ and $k$, which are exclusively bound to regular and interstitial lattice, are represented by $v_{\text {sub:i }}^{\alpha}$ and $v_{\text {ints: } k}^{\alpha}$, respectively, while $N_{\text {sub: } i}^{\alpha}$ and $N_{\text {ints: } k}^{\alpha}$ correspond to the number of moles of these chemical species. Given the nature of the interstitial sites, it is reasonably assumed that the components occupying this lattice marginally contribute to the volume. Additionally, it is considered that volume of phase- $\alpha$ is identically influenced by all the chemical species that are confined to the regular lattice. These assumptions, in terms of the partial molar-volumes, read

$$
\begin{aligned}
& v_{\text {ints:1 }}^{\alpha}=v_{\text {ints:2 }}^{\alpha}=\cdots=v_{\text {ints:l }}^{\alpha} \equiv v_{\text {ints }}^{\alpha}=0, \quad \text { and } \\
& v_{\text {sub:1 }}^{\alpha}=v_{\text {sub:2 }}^{\alpha}=\cdots=v_{\text {sub:j } j}^{\alpha} \equiv v_{\text {sub }}^{\alpha} .
\end{aligned}
$$

By imposing the above approximations in Eqn. (14), a summation constraint, written as

$$
\sum_{i=1}^{j} \frac{N_{\text {sub: } i}^{\alpha}}{V^{\alpha}} \equiv \sum_{i=1}^{j} \rho_{\text {sub: } i}^{\alpha}=\frac{1}{v_{\text {sub }}^{\alpha}},
$$


can be exclusively derived for the components occupying regular lattice. The individual entities of the summation series in Eqn. (16) correspond to the number of moles of a regular-lattice component per unit volume of phase- $\alpha, \frac{N_{\text {sub:i }}^{\alpha}}{V^{\alpha}}$, which is referred to as the molar number-density, $\rho_{\text {sub: } i}^{\alpha}$. Corollary of Eqns. (15) and (16) is that number-density, when adopted to describe the chemical composition of a phase, inherently distinguishes components occupying regular and interstitial lattice.

Considering the description of the mole fraction of component- $i$ in phase- $\alpha$, which contains $j$ and $l$ chemical species respectively bound to regular and interstitial sites, $c_{\text {sub: } i}^{\alpha}$ associated with regular lattice can be related to the corresponding number-density as

$$
c_{\mathrm{sub}: i}^{\alpha}=\left[\frac{c_{\mathrm{sub}: i}^{\alpha}}{V^{\alpha}}\right]\left[\frac{V^{\alpha}}{\sum_{i=1}^{j} c_{\mathrm{sub}: i}^{\alpha}+\sum_{k=1}^{l} c_{\mathrm{ints}: i}^{\alpha}}\right]=\rho_{\mathrm{sub}: i}^{\alpha} V_{m}^{\alpha},
$$

where $V_{m}^{\alpha}$ molar of phase- $\alpha$ [28]. Analogous expression can be derived for interstitial components, that reads

$$
\rho_{\text {ints: } k}^{\alpha}=\frac{c_{\text {ints: } k}^{\alpha}}{V_{m}^{\alpha}},
$$

where $\rho_{\text {ints: } k}^{\alpha}$ and $c_{\text {ints: } k}$ correspond to the number-density and mole fraction of component- $k$ occupying interstitial site.

Despite being occupied by certain alloying elements, generally, interstitial lattice include considerable amount of vacant sites. Since the vacant sites are not encompassed in the formulation of mole fraction, the resulting relation in Eqn. (18) primarily considers the interstitial sites that are occupied. For a given volume of phase- $\alpha$, the number-density of the overall interstitial sites including vacant ones are constant, and can be written as

$$
S_{\text {ints }}^{\alpha}=\sum_{k=1}^{l} \rho_{\text {ints: } k}^{\alpha}+\rho_{\text {ints:Va }}^{\alpha},
$$

where $\rho_{\text {ints:Va }}^{\alpha}$ is the number-density of vacant interstitial site at a given instant of the transformation. The overall number-density of the interstitial site, $S_{\text {ints }}^{\alpha}$, is material-specific and is considerably influenced by crystal structure. In view of the overall interstitial number-density, Eqn. (18) can be re-formulated as

$$
\rho_{\text {ints: } k}^{\alpha}=c_{\text {ints: } k}^{\alpha}\left[S_{\text {ints }}^{\alpha}+\frac{1}{v_{\text {sub }}^{\alpha}}-\rho_{\text {ints:Va }}^{\alpha}\right] \text {, }
$$


wherein number-density of the vacant interstitial sites are included.

While separately emphasising the significance of molar- and partial molar-volume in the current formulation, Eqns. (16) and (17) indicate that a relation between these parameters can be derived. Such relation, as will be shown in the subsequent sections, facilitates a rather straightforward description of the free-energy densities, considering that the driving force should be expressed based on the currently adopted concentration variable, i.e, molar number-density.

A condition associating molar and partial molar volume can be derived from any formulation that delineate the concentration of regular or interstitial components. For instance, exclusively considering the components bound to regular lattice, the summation of their concentration, based on Eqn. (17), is written as

$$
\sum_{i=1}^{j} \rho_{\text {sub:i }}^{\alpha}=\frac{1}{V_{m}^{\alpha}} \sum_{i=1}^{j} c_{\text {sub: } i}^{\alpha} .
$$

In a phase comprising of chemical species occupying interstitial sites, owing to the definition of mole fraction, summation on the right side of the above Eqn. (21) always follows $\sum_{i=1}^{j} c_{\text {sub:i }}^{\alpha}<1$. Correspondingly, by substituting Eqn. (16), a condition relating molar and partial molar can be written as

$$
V_{m}^{\alpha} \leq v_{\text {sub }}^{\alpha}
$$

The role of Eqn. (16) in deriving the above relation entails that Eqn. (22) is valid only when the components occupying regular lattice render an identical contribution to the volume (Eqn. (15)).

In the absence of any interstitial chemical species, Eqn. (22) indicates that the molar and partial molar volume are equal. Moreover, for a dilute system with a negligible quantity of components occupy interstitial sites, the volume parameters can be approximated as $V_{m}^{\alpha} \approx v_{\text {sub }}^{\alpha}$.

\subsection{Re-formulated grand-potential model}

Adopting molar number-density, a multicomponent grand-potential phase-field model is formulated to distinctly realise substitution and interstitial diffusion. As elucidated in the previous section, expressing the composition of a system in number-density inherently distinguishes components occupying regular and interstitial lattices. This distinction rendered by molar numberdensity is exploited to differentiate the varying modes of diffusion. 


\subsubsection{Grand-potential density}

A two-phase multicomponent system with $m$ chemical species is considered for re-formulating the existing grand-potential model. Owing to the constraint $\phi_{\alpha}+\phi_{\beta}=1$, the system is adequately defined by a scalar phase-field $(\phi)$. Furthermore, $m$ chemical species are distinguished into $j$ and $l$ components, $m \ni\{$ sub: 1 , sub: $2, \ldots$, sub: $j$, ints: 1 , ints: $2, \ldots$, ints: $l\}$, depending on the lattice sites occupied by each of these species. While the component 'sub: $i$ ' is bound to the regular lattice, and diffuses substitutionally, interstitially migrating chemical species are represented by 'ints:l'.

The overall chemical composition of a phase- $\alpha$, for the current consideration, is described by a tuple comprising of $m$-entities, which is written as

$$
\boldsymbol{\rho}^{\alpha} \equiv\{\underbrace{\rho_{\text {sub:1 }}^{\alpha}, \rho_{\text {sub:2 }}^{\alpha}, \ldots, \rho_{\text {sub: }:}^{\alpha}}_{:=\boldsymbol{\rho}_{\text {sub }}^{\alpha}}, \underbrace{\rho_{\text {ints:1 }}^{\alpha}, \rho_{\text {ints:2 }}^{\alpha}, \ldots, \rho_{\text {ints: }: k}^{\alpha}}_{:=\boldsymbol{\rho}_{\text {ints }}^{\alpha}}\},
$$

where $\rho_{\text {sub:j }}^{\alpha}$ and $\rho_{\text {ints: } k}^{\alpha}$ correspond to the number-density based concentration of species- $j$ and $-k$ respectively occupying regular and interstitial sites. The continuous variable associated with each of these components are analogously represented by

$$
\boldsymbol{\mu} \equiv\{\underbrace{\mu_{\text {sub:2 }}, \mu_{\text {sub:3 } 3, \ldots, \mu_{\text {sub: }:}}}_{\boldsymbol{\mu}_{\text {sub }}}, \underbrace{\mu_{\text {ints: } 1,}, \mu_{\text {ints:2 }}, \ldots, \mu_{\text {ints: }: k}}_{\boldsymbol{\mu}_{\text {ints }}}\}
$$

with $\mu_{\text {sub: } j}$ and $\mu_{\text {ints: } k}$ indicating diffusion and chemical potential of components- $j$ and $-k$, respectively. Diffusion and chemical potential are appropriately related to components occupying regular and interstitial sites through proper formulation of the free-energy densities.

For consistent formulation, molar free-energies $\left(\mathrm{J} \mathrm{mol}^{-1}\right)$ of individual phases, which are often rendered by databases like CALPHAD, are generally converted to energy densities $\left(\mathrm{J} \mathrm{m}^{-3}\right)$ through

$$
f^{\alpha}\left(\boldsymbol{c}^{\alpha}\right)=\frac{1}{V_{m}} G^{\alpha}\left(\boldsymbol{c}^{\alpha}\right)
$$

where $\boldsymbol{c}^{\alpha}$ encompasses concentration of $m$ components in mole fraction [28, 30]. This conversion from Gibbs, $G^{\alpha}\left(\boldsymbol{c}^{\alpha}\right)$, to Helmholtz energy-density, $f^{\alpha}\left(\boldsymbol{c}^{\alpha}\right)$, is achieved by assuming that molar volume of all constituent phases are equal $\left(V_{m}^{\alpha}=V_{m}^{\beta}=V_{m}\right)$. Using conjugate pairs, and 
differentiating components based on their lattice positions, Eqn. 25 can be written as

$$
f^{\alpha}\left(\boldsymbol{c}^{\alpha}\right)=\frac{1}{V_{m}}\left(\sum_{i=1}^{j} \tilde{\mu}_{\text {sub:i }}^{\alpha} c_{\text {sub: } i}^{\alpha}+\sum_{k=1}^{l} \mu_{\text {ints: } k}^{\alpha} c_{\text {ints: } k}^{\alpha}\right)
$$

where $c_{\text {sub:i }}^{\alpha}$ and $c_{\text {ints:i }}^{\alpha}$ is the mole fraction of species $-i$ and $-k$ [31]. Moreover, chemical potential of component $-i$ and $-k$ are respectively denoted by $\tilde{\mu}_{\text {sub: } i}$ and $\mu_{\text {ints: } k}^{\alpha}$. By substituting Eqns. (17) and (18), the free-energy density of phase- $\alpha$, in terms of molar number-density, is expressed as

$$
f^{\alpha}\left(\boldsymbol{\rho}^{\alpha}\right)=\sum_{i=1}^{j} \tilde{\mu}_{\text {sub:i }}^{\alpha} \rho_{\text {sub: } i}^{\alpha}+\sum_{k=1}^{l} \mu_{\text {ints: } k}^{\alpha} \rho_{\text {ints: } k}^{\alpha} .
$$

When the conjugate pair of the solvent-matrix are separated from the alloying elements, the above Eqn. 27) transforms to

$$
f^{\alpha}\left(\boldsymbol{\rho}^{\alpha}\right)=\tilde{\mu}_{\text {sub:1 }}^{\alpha} \rho_{\text {sub: } 1}^{\alpha}+\sum_{i=2}^{j} \tilde{\mu}_{\text {sub: }:}^{\alpha} \rho_{\text {sub: } i}^{\alpha}+\sum_{k=1}^{l} \mu_{\text {ints: } k}^{\alpha} \rho_{\text {ints: } k}^{\alpha},
$$

where $\rho_{\text {sub:1 }}^{\alpha}$ and $\tilde{\mu}_{\text {sub:1 }}^{\alpha}$ are the concentration of solvent in number-density, and its corresponding chemical potential. By imposing the summation constraint associated with concentration of components occupying regular lattice, Eqn. (16), the number-density of the solvent-matrix is expanded, and the resulting free-energy formulation is written as

$$
f^{\alpha}\left(\boldsymbol{\rho}^{\alpha}\right)=\tilde{\mu}_{\text {sub:1 }}^{\alpha}\left(\frac{1}{v_{\text {sub }}^{\alpha}}-\sum_{i=2}^{j} \tilde{\mu}_{\text {sub: } i}^{\alpha} \rho_{\text {sub: } i}^{\alpha}\right)+\sum_{i=2}^{j} \tilde{\mu}_{\text {sub: } i}^{\alpha} \rho_{\text {sub:i }}^{\alpha}+\sum_{k=1}^{l} \mu_{\text {ints: } k}^{\alpha} \rho_{\text {ints: } k}^{\alpha}
$$

Re-arranging the terms in the above expression yields

$$
f^{\alpha}\left(\boldsymbol{\rho}^{\alpha}\right)=\frac{\tilde{\mu}_{\text {sub: } 1}^{\alpha}}{v_{\text {sub }}^{\alpha}}+\sum_{i=2}^{j} \mu_{\text {sub:i }}^{\alpha} \rho_{\text {sub: } i}^{\alpha}+\sum_{k=1}^{l} \mu_{\text {ints: } k}^{\alpha} \rho_{\text {ints: } k}^{\alpha},
$$

where $\mu_{\text {sub: } i}^{\alpha}=\tilde{\mu}_{\text {sub: } i}^{\alpha}-\tilde{\mu}_{\text {sub: } 1}^{\alpha}$ is the diffusion potential of chemical species- $i$ bound to the regular lattice site. This formulation reduces the number of independent variable to $m-1$ through the summation constraint associated regular-lattice components (Eqn. (16)). It should be noted that similar operation cannot be extended for the interstitial components, since from the very outset the expression of the concentration in mole fraction does not include vacant sites. Therefore, consistent with the thermodynamic description, the free-energy density in Eqn. 30 is formulated based on the diffusion and chemical potential of regular-lattice and interstitial components, 
respectively [34]. However, the number of independent variables can further be decreased when the concentration is originally expressed in site fractions, but given that the chemical potential of vacant site is negligible, in Eqn. (30) the conjugate pair of the interstitial concentration would continue to be chemical potential, not diffusion potential [32].

Bulk contribution of a given phase in grand-potential based phase-field model is described by the Legendre transform of its free-energy density [33]. Therefore, the grand-potential density of phase- $\alpha$, based on Eqn. (30), is expressed as

$$
\psi_{\alpha}\left(\boldsymbol{\rho}^{\alpha}(\boldsymbol{\mu})\right)=f^{\alpha}\left(\boldsymbol{\rho}^{\alpha}\right)-\sum_{i=2}^{j} \mu_{\text {sub:i }} \rho_{\text {sub:i }}^{\alpha}-\sum_{k=1}^{l} \mu_{\text {ints: } k} \rho_{\text {ints: } k}^{\alpha} .
$$

wherein, owing to the local-equilibrium consideration, the diffusion and chemical potential of the respective regular and interstitial lattice components are treated as phase-independent continuous variables. In other words, as indicated in Eqn. (24), through proper description of the free-energy density, appropriate potentials are consistently treated as the continuous variable for components bound to regular and interstitial sites [34]. Furthermore, given the local-equilibrium condition $\left(\tilde{\mu}_{\text {sub:1 }}^{\alpha}=\tilde{\mu}_{\text {sub:1 }}^{\beta}\right)$ and the assumption, $v_{\text {sub }}^{\alpha}=v_{\text {sub }}^{\beta} \equiv v_{\text {sub }}$, any contribution from the solvent-matrix in Eqn. (30), $\frac{\tilde{\mu}_{\text {sub:1 }}^{\alpha}}{v_{\text {sub }}^{\alpha}}$, to the driving force is annulled.

The functional detailing the energy-density of the entire system of volume $V$ is written as

$$
\Omega(\boldsymbol{\mu}, \phi, \boldsymbol{\nabla} \phi)=\int_{V} \mathrm{~d} V\left[\psi\left(\boldsymbol{\rho}^{\alpha}(\boldsymbol{\mu}), \boldsymbol{\rho}^{\beta}(\boldsymbol{\mu}), \phi\right)+\frac{1}{\varepsilon} f_{\mathrm{pen}}(\phi)+\varepsilon \gamma|\boldsymbol{\nabla} \phi|^{2}\right],
$$

where $\psi\left(\boldsymbol{\rho}^{\alpha}(\boldsymbol{\mu}), \boldsymbol{\rho}^{\beta}(\boldsymbol{\mu}), \phi\right)$ is the overall grand-potential density which is defined by interpolating the bulk contribution of the individual phases in Eqn. (31] [35]. An interpolation function of the form $h(\phi)=\phi^{2}(3-2 \phi)$ is adopted in the present work. Moreover, a conventional doublewell type potential, $f_{\text {pen }}(\phi)=\frac{16}{\pi^{2}} \gamma \phi^{2}(1-\phi)^{2}$, is used as a penalising function in Eqn. (32). The length scale-parameter $\varepsilon$ and $\gamma$ respectively dictate the width and energy-density of the interface separating the phases [36].

\subsubsection{Evolution equation}

Microstructural transformation of the system is modelled by considering a phenomenological decrease in its overall energy-density. Accordingly, in the current two-phase grand potential 
framework, the temporal evolution of phase-field that translates into the observed microstructural changes is formulated as

$$
\begin{aligned}
\tau \varepsilon \frac{\partial \phi}{\partial t} & =-\frac{\delta \Omega(\boldsymbol{\mu}, \phi, \boldsymbol{\nabla} \phi)}{\delta \phi} \\
& =2 \gamma \varepsilon \boldsymbol{\nabla}^{2} \phi-\frac{1}{\varepsilon} \frac{\partial f_{\text {pen }}(\phi)}{\partial \phi}-\underbrace{\left[\psi_{\alpha}(\boldsymbol{\mu})-\psi_{\beta}(\boldsymbol{\mu})\right]}_{\Delta \psi(\boldsymbol{\mu})} \frac{\partial h(\phi)}{\partial \phi},
\end{aligned}
$$

where $\tau$ is the relaxation parameter that ensures stability of the diffuse interface, and $\varepsilon$ denotes a length-scale parameter dictating the width of the interface. It is evident from the above evolution equation that, in the grand-potential formalism, the bulk driving-force, which dictates that the spatio-temporal change in phase-field, corresponds to the difference in the grand-potential densities of individual phases $(\Delta \psi(\boldsymbol{\mu}))$.

The temporal change in the continuous variable, that influences the observed microstructural transformation through the bulk driving-force, is derived by considering the evolution of the concentration $(\boldsymbol{\rho})$ and its associated variables $(\boldsymbol{\mu}, \phi)$ [37]. As delineated in previous section, the continuous variables are characteristically different for components occupying regular and interstitial sites. In other words, while diffusion potential predominantly governs the migration of regular-lattice chemical species $\left(\boldsymbol{\mu}_{\text {sub }}\right)$, interstitial diffusion of the corresponding alloying elements is dictated by chemical potential $\left(\boldsymbol{\mu}_{\text {ints }}\right)$. Despite the fundamental disparity, the temporal evolution of the continuous variable(s) is expressed in an unified manner as

$$
\frac{\partial \mu_{i}}{\partial t}=[\underbrace{\boldsymbol{\chi}_{i k}^{\alpha} h(\phi)+\boldsymbol{\chi}_{i k}^{\beta}(1-h(\phi))}_{:=\boldsymbol{\chi}(\phi)}]^{-1}[\underbrace{\boldsymbol{\nabla} \cdot \sum_{i=1}^{m-1} \boldsymbol{M}_{i k}(\phi) \boldsymbol{\nabla} \mu_{k}}_{:=\frac{\partial \rho_{i}}{\partial t}}-\left(\rho_{i}^{\alpha}-\rho_{i}^{\beta}\right) \frac{\partial h(\phi)}{\partial t}],
$$

where $i \in\{$ sub: 1, sub:2, 2, sub: $j$, ints: 1 , ints: $2, \ldots$, ints: $l\}$, and $m(=j+l)$ encompasses all components irrespective of its lattice positions.

The mobility of components in Eqn. (34) is governed by $\boldsymbol{M}_{i k}(\phi)$, which is written as

$$
\boldsymbol{M}_{i k}(\phi)=\boldsymbol{D}_{i k}^{\alpha} \boldsymbol{\chi}_{i k}^{\alpha} h(\phi)+\boldsymbol{D}_{i k}^{\beta} \boldsymbol{\chi}_{i k}^{\beta}(1-h(\phi))
$$

with $\boldsymbol{D}_{i k}^{\alpha}$ and $\chi_{i k}^{\beta}$ representing the interdiffusivity and susceptibility matrix. The formulation of 
interdiffusivity matrix of dimension $[m-1 \times m-1]$ as

$$
\begin{aligned}
& \boldsymbol{D}_{i k}^{\alpha}=\left(\begin{array}{ccc|ccc}
D_{\text {sub }_{2} \text { sub }_{2}}^{\alpha} & \ldots & D_{\text {sub }_{2} \text { sub }_{j}}^{\alpha} & D_{\text {sub }_{2} \text { ints }_{1}}^{\alpha} & \ldots & D_{\text {sub }_{2} \text { ints }_{l}}^{\alpha} \\
D_{\text {sub }_{3} \text { sub }_{2}}^{\alpha} & \ldots & D_{\text {sub }_{3} \text { sub }_{j}}^{\alpha} & D_{\text {sub }_{3} \text { ints }_{1}}^{\alpha} & \ldots & D_{\text {sub }_{3} \text { ints }_{l}}^{\alpha} \\
\vdots & \ddots & \vdots & \vdots & \ddots & \vdots \\
D_{\text {sub }_{j} \text { sub }_{2}}^{\alpha} & \ldots & D_{\text {sub }_{j} \text { sub }_{j}}^{\alpha} & D_{\text {sub }_{j} \text { ints }_{1}}^{\alpha} & \ldots & D_{\text {sub }_{j} \text { ints }_{l}}^{\alpha} \\
\hline D_{\text {ints }_{1} \text { sub }_{2}}^{\alpha} & \ldots & D_{\text {ints }_{1} \text { sub }_{j}}^{\alpha} & D_{\text {ints }_{1} \text { ints }_{1}}^{\alpha} & \ldots & D_{\text {ints }_{1} \text { ints }_{l}}^{\alpha} \\
D_{\text {ints }_{2} \text { sub }_{2}}^{\alpha} & \ldots & D_{\text {ints }_{2} \text { sub }_{j}}^{\alpha} & D_{\text {ints }_{2} \text { ints }_{1}}^{\alpha} & \ldots & D_{\text {ints }_{2} \text { ints }_{l}}^{\alpha} \\
\vdots & \ddots & \vdots & \vdots & \ddots & \vdots \\
D_{\text {ints }_{l} \text { sub }_{2}}^{\alpha} & \ldots & D_{\text {ints }_{l} \text { sub }_{j}}^{\alpha} & D_{\text {ints }_{l} \text { ints }_{1}}^{\alpha} & \ldots & D_{\text {ints }_{l} \text { ints }_{l}}^{\alpha}
\end{array}\right) \\
& =\left(\begin{array}{c|c}
\boldsymbol{D}_{\text {sub }}^{\alpha} & \boldsymbol{D}_{\text {sub:ints }}^{\alpha} \\
\hline \boldsymbol{D}_{\text {sub:ints }}^{\alpha} & \boldsymbol{D}_{\text {ints }}^{\alpha}
\end{array}\right),
\end{aligned}
$$

and analogous description of susceptibility matrix, lends itself to the unified expression and implementation of continuous variable(s) evolution.

\section{Incorporation quantitative driving force}

A wide-range of approaches are adopted to incorporate CALPHAD-based quantitative drivingforce in phase-field formalism. These include extending the numerical framework of the model by appending datasheet that comprises of essential information [38], coupling locally-linearised phase diagrams [39], and parallely running the associated software packages [40]. In addition to the aforementioned techniques, appropriate bulk contribution has been introduced by approximating the Gibbs free energy as a second-order function [41]. Owing to the elagancy of the formulation, and its ability to render consistent driving-force, CALPHAD data is incorporated through parabolic approximation of free-energy density in present work. 


\subsection{Generalised formulation}

Free-energy density of a phase consisting of $m$ chemical species, at a given temperature $T$, can be expanded around its equilibrium composition as

$$
f^{\Theta}(\boldsymbol{c})=\frac{1}{V_{m}}\left\{G^{\Theta}\left(\boldsymbol{c}_{\mathrm{eq}}^{\Theta}\right)+\sum_{i=1}^{m}\left[\frac{\partial G^{\Theta}(\boldsymbol{c})}{\partial c_{i}}\right]_{c_{i: \mathrm{eq}}^{\Theta}}\left(c_{i}-c_{i \text { :eq }}^{\Theta}\right)+\frac{1}{2} \sum_{i \leq j}\left[\frac{\partial^{2} G^{\Theta}(\boldsymbol{c})}{\partial c_{i} \partial c_{j}}\right]_{c_{\mathrm{eq}}^{\Theta}}\left(c_{i}-c_{i: \mathrm{eq}}^{\Theta}\right)\left(c_{j}-c_{j \text { :eq }}^{\Theta}\right)\right\},
$$

where $\Theta \in\{\alpha, \beta\}$, and $G^{\Theta}(\boldsymbol{c})$ denotes its Gibbs free-energy [42]. While $\boldsymbol{c}_{\mathrm{eq}}^{\Theta}$ encompasses of equilibrium concentration of all chemical species in phase- $\Theta, c_{i: \text { eq }}^{\Theta}$ and $c_{j \text { :eq }}^{\Theta}$ correspond to the equilibrium composition of component $i$ and $j$. Moreover, in Eqn. (37), $V_{m}$ represents identical molar volume of phase $-\alpha$ and $-\beta\left(V_{m}^{\alpha}=V_{m}^{\beta}=V_{m}\right)$.

Since Eqn. (37) reflects the data rendered by CALPHAD, the concentration is treated in mole fraction. Re-writing the concentration in number density based on Eqn. (17), the resulting expression for free-energy density reads

$f^{\Theta}(\boldsymbol{\rho})=f^{\Theta}\left(\boldsymbol{\rho}_{\text {eq }}^{\Theta}\right)+\sum_{i=1}^{m}\left[\frac{\partial G^{\Theta}(\boldsymbol{\rho})}{\partial \rho_{i}}\right]_{\rho_{i: \mathrm{eq}}^{\Theta}}\left(\rho_{i}-\rho_{i: \mathrm{eq}}^{\Theta}\right)+\frac{V_{m}}{2} \sum_{i \leq j}\left[\frac{\partial^{2} G^{\Theta}(\boldsymbol{\rho})}{\partial \rho_{i} \partial \rho_{j}}\right]_{\rho_{\text {eq }}^{\Theta}}\left(\rho_{i}-\rho_{i: \mathrm{eq}}^{\Theta}\right)\left(\rho_{j}-\rho_{j: \mathrm{eq}}^{\Theta}\right)$,

where $\boldsymbol{\rho}_{\text {eq }}^{\Theta}$, which includes $\rho_{i \text { :eq }}^{\Theta}$ and $\rho_{j \text { :eq }}^{\Theta}$, indicates the equilibrium concentration in molar numberdensity. As delineated in previous sections, $m$ components constituting phase- $\Theta$ can be distinguished based on their lattice positions. Correspondingly, by segregating the contribution of the chemical species occupying regular lattice positions from the ones confined to the interstitial 
sites, the free-energy density is written as

$$
\begin{aligned}
& f^{\Theta}(\boldsymbol{\rho})=f^{\Theta}\left(\boldsymbol{\rho}_{\text {eq }}^{\Theta}\right)+\sum_{i=1}^{j}\left[\frac{\partial G^{\Theta}(\boldsymbol{\rho})}{\partial \rho_{\text {sub: } i}}\right]_{\rho_{\text {eq }}^{\Theta}}\left(\rho_{\text {sub: } i}-\rho_{\text {sub: } i_{\text {eq }}}^{\Theta}\right)+\sum_{k=1}^{l}\left[\frac{\partial G^{\Theta}(\boldsymbol{\rho})}{\partial \rho_{\text {ints: } k}}\right]_{\rho_{\text {eq }}^{\Theta}}\left(\rho_{\text {ints: } k}-\rho_{\text {ints: } k_{\text {eq }}}^{\Theta}\right) \\
& +\frac{V_{m}}{2} \sum_{i \leq j}\left[\frac{\partial^{2} G^{\Theta}(\boldsymbol{\rho})}{\partial \rho_{\text {sub: } i} \partial \rho_{\text {sub: } j}}\right]_{\rho_{\mathrm{eq}}^{\Theta}}\left(\rho_{\text {sub: } i}-\rho_{\text {sub: } i_{\mathrm{eq}}}^{\Theta}\right)\left(\rho_{\text {sub: } j}-\rho_{\text {sub: } j_{\text {eq }}}^{\Theta}\right) \\
& +\frac{V_{m}}{2} \sum_{k \leq l}\left[\frac{\partial^{2} G^{\Theta}(\boldsymbol{\rho})}{\partial \rho_{\text {ints: } k} \partial \rho_{\text {ints:l }}}\right]_{\rho_{\text {eq }}^{\Theta}}\left(\rho_{\text {ints: } k}-\rho_{\text {ints: } k_{\text {eq }}}^{\Theta}\right)\left(\rho_{\text {ints:l }}-\rho_{\text {ints: } l_{\text {eq }}}^{\Theta}\right) \\
& +\frac{V_{m}}{2} \sum_{i \neq k}\left[\frac{\partial^{2} G^{\Theta}(\boldsymbol{\rho})}{\partial \rho_{\text {sub: } i} \partial \rho_{\text {ints: } k}}\right]_{\rho_{\mathrm{eq}}^{\Theta}}\left(\rho_{\text {sub: } i}-\rho_{\text {sub: } i_{\mathrm{eq}}}^{\Theta}\right)\left(\rho_{\text {ints: } k}-\rho_{\text {ints: } k_{\mathrm{eq}}}^{\Theta}\right) .
\end{aligned}
$$

The second derivative of the Gibbs free energy involved in above Eqn. (39) effects the mobility of the continuous variable, and can be extracted from kinetic databases.

Legendre transform of free-energy density of a phase yields its corresponding grand-potential density [21]. Accordingly, grand-potential density of phase- $\alpha$, analogous to Eqn. (39), is expressed as

$$
\begin{aligned}
\psi^{\Theta}(\boldsymbol{\mu})=\psi^{\Theta}\left(\boldsymbol{\mu}_{\mathrm{eq}}\right) & +\sum_{i=1}^{j}\left[\frac{\partial \psi^{\Theta}(\boldsymbol{\mu})}{\partial \tilde{\mu}_{\text {sub: } i}}\right]_{\boldsymbol{\mu}_{\mathrm{eq}}}\left(\tilde{\mu}_{\text {sub:i}}-\tilde{\mu}_{\text {sub: } i_{\mathrm{eq}}}\right)+\sum_{k=1}^{l}\left[\frac{\partial \psi^{\Theta}(\boldsymbol{\mu})}{\partial \mu_{\mathrm{ints}: k}}\right]_{\boldsymbol{\mu}_{\mathrm{eq}}}\left(\mu_{\mathrm{ints}: k}-\mu_{\mathrm{ints}: k_{\mathrm{eq}}}\right) \\
& -\frac{V_{m}}{2} \sum_{i \leq k}^{m, m} \boldsymbol{\chi}_{i k}^{\Theta}\left(\tilde{\mu}_{\text {sub: } i}-\tilde{\mu}_{\text {sub: } i_{\mathrm{eq}}}\right)\left(\mu_{\text {ints: } k}-\mu_{\text {ints: } k_{\mathrm{eq}}}\right)
\end{aligned}
$$

where $\chi_{i k}^{\Theta}$ is the susceptibility matrix adopting the form of interdiffusivity matrix $\boldsymbol{D}_{i k}^{\alpha}$ in Eqn. (36), wherein entities of this tensor are reciprocal of second derivative of Gibbs free energy [42, 21]. Moreover, in Eqn. (40), it is important to re-iterate that $\tilde{\mu}_{\text {sub: } i}$ and $\mu_{\text {ints: } k}$ denote the chemical potential of component $-i$ and $-k$, which respectively occupy regular and interstitial lattice-sites. Owing to its continuous nature in the current formulation, chemical potentials are indicated as phase-independent variables. The equilibrium chemical potential of species- $i$ and $-k$, which are to bound to regular and interstitial lattice, are respectively denoted by $\tilde{\mu}_{\text {sub: } i_{\mathrm{eq}}}$ and $\mu_{\text {ints: } k_{\mathrm{eq}}}$. By imposing the relation

$$
\frac{\partial \psi^{\Theta}(\boldsymbol{\mu})}{\partial \mu_{i}}=-\rho_{i}^{\Theta}
$$


for a generic component- $i$ on Eqn. (40), grand-potential density of phase- $\Theta$ can be written as

$$
\begin{aligned}
\psi^{\Theta}(\boldsymbol{\mu})=\psi^{\Theta}\left(\boldsymbol{\mu}_{\mathrm{eq}}\right) & -\sum_{i=1}^{j} \rho_{\text {sub: } i_{\mathrm{eq}}}^{\Theta}\left(\tilde{\mu}_{\text {sub: } i}-\tilde{\mu}_{\text {sub: } i_{\mathrm{eq}}}\right)-\sum_{k=1}^{l} \rho_{\text {ints: } k_{\mathrm{eq}}}^{\Theta}\left(\mu_{\text {ints: } k}-\mu_{\text {ints: } k_{\mathrm{eq}}}\right) \\
& -\frac{V_{m}}{2} \sum_{i \leq k}^{m, m} \boldsymbol{\chi}_{i k}^{\Theta}\left(\tilde{\mu}_{\text {sub: } i}-\tilde{\mu}_{\text {sub: } i_{\mathrm{eq}}}\right)\left(\mu_{\text {ints: } k}-\mu_{\text {ints: } k_{\mathrm{eq}}}\right),
\end{aligned}
$$

where $\rho_{\text {sub: } i_{\text {eq }}}^{\Theta}$ and $\rho_{\text {ints: } k_{\text {eq }}}^{\Theta}$ correspond to the number-density based equilibrium concentration of component- $i$ and $-k$ occupying regular and interstitial lattice sites in phase- $\Theta$. In order to express the contribution of the regular-lattice components in terms of thermodynamically-consistent diffusion potential, the associated summation constraint (Eqn. (16) is introduced in the formulation of grand-potential density. Accordingly, the grand-potential density of phase- $\Theta$ in Eqn. (42) is re-formulated as

$$
\begin{aligned}
\psi^{\Theta}(\boldsymbol{\mu})=\psi^{\Theta}\left(\boldsymbol{\mu}_{\mathrm{eq}}\right) & -\frac{1}{v_{\text {sub }}^{\Theta}}\left(\tilde{\mu}_{\text {sub: } 1}-\tilde{\mu}_{\text {sub: } 1_{\mathrm{eq}}}\right)-\sum_{i=2}^{j} \rho_{\text {sub: } i_{\mathrm{eq}}}^{\Theta}\left(\mu_{\text {sub: } i}-\mu_{\text {sub: } i_{\mathrm{eq}}}\right) \\
& -\sum_{k=1}^{l} \rho_{\text {ints: } k_{\mathrm{eq}}}^{\Theta}\left(\mu_{\text {ints: } k}-\mu_{\text {ints: } k_{\mathrm{eq}}}\right)-\frac{V_{m}}{2} \sum_{i \leq k}^{m, m} \chi_{i k}^{\Theta}\left(\tilde{\mu}_{\text {sub: } i}-\tilde{\mu}_{\text {sub }: i_{\mathrm{eq}}}\right)\left(\mu_{\text {ints: } k}-\mu_{\text {ints: } k_{\mathrm{eq}}}\right),
\end{aligned}
$$

where $\mu_{\text {sub:i } i}$ and $\mu_{\text {sub: } i_{\mathrm{eq}}}$ are diffusion potential and its equilibrium counterpart of chemical species$i$, respectively. In Eqn. 43, $\tilde{\mu}_{\text {sub:1 }}$ and $\tilde{\mu}_{\text {sub: } 1_{\mathrm{eq}}}$ represents the chemical potentials of solvent-matrix, while $v_{\text {sub }}^{\Theta}$ is partial molar-volume which is assumed to be equal for all components bound to regular lattice.

In grand-potential based phase-field model, as indicated in Eqn. (33), the bulk driving-force is formulated as the difference in the potential densities of individual phases. Therefore, the driving-force governing phase-field evolution in present model, wherein the energy densities are approximated as second-degree polynomials, is expressed as

$$
\begin{aligned}
\Delta \psi^{\alpha \beta}(\boldsymbol{\mu}) & =\psi^{\alpha}(\boldsymbol{\mu})-\psi^{\beta}(\boldsymbol{\mu}) \\
& =\sum_{i=2}^{j}\left(\rho_{\text {sub: } i_{\mathrm{eq}}}^{\beta}-\rho_{\text {sub: } i_{\mathrm{eq}}}^{\alpha}\right)\left(\mu_{\text {sub: } i}-\mu_{\text {sub: } i_{\mathrm{eq}}}\right)+\sum_{k=1}^{l}\left(\rho_{\text {ints: } k_{\mathrm{eq}}}^{\beta}-\rho_{\text {ints: } k_{\mathrm{eq}}}^{\alpha}\right)\left(\mu_{\text {ints: } i}-\mu_{\text {ints: } i_{\mathrm{eq}}}\right) \\
& +\frac{V_{m}}{2} \sum_{i \leq k}^{m, m}\left(\boldsymbol{\chi}_{i k}^{\beta}-\boldsymbol{\chi}_{i k}^{\alpha}\right)\left(\mu_{\text {sub:i }}-\mu_{\text {sub: }: \text { eq }_{\mathrm{eq}}}\right)\left(\mu_{\text {ints:i } i}-\mu_{\text {ints: } i_{\mathrm{eq}}}\right) .
\end{aligned}
$$


The above description of the driving force ensues from the assumption that partial molar-volume is equal across the phases $\left(v_{\text {sub }}^{\alpha}=v_{\text {sub }}^{\beta} \equiv v_{\text {sub }}\right.$ ), which eliminates the contribution of solventmatrix. Moreover, considering that diffusion potential is the difference in the chemical potential of a component and its solvent matrix, $\left(\tilde{\mu}_{\text {sub: } i}-\tilde{\mu}_{\text {sub: } i_{\mathrm{eq}}}\right)$ is replaced by $\left(\mu_{\text {sub: } i}-\mu_{\text {sub: } i_{\mathrm{eq}}}\right)$ in Eqn. (44). Since grand-potential densities of phase- $\alpha$ and $-\beta$ are equal at equilibrium, $\psi^{\alpha}\left(\boldsymbol{\mu}_{\mathrm{eq}}\right)=\psi^{\beta}\left(\boldsymbol{\mu}_{\mathrm{eq}}\right)$, the driving force is primarily dictated by the deviation from equilibrium chemical and diffusion potential. The formulation of driving force in Eqn. (44) ensures that, while chemical potential of interstitial components contribute to driving force, it is diffusion potential of regular-lattice species that renders analogous contribution.

\subsection{Ternary Fe - Mn - C system}

Ability of the current re-formulated approach to distinguish interstitial and substitutional diffusion, and to model a corresponding phase transformation in system comprising of components exclusively bound to regular and interstitial lattice is primarily discussed in view of manganese steels. Following the generalised approximation delineated in previous section, the CALPHADbased free energy-density of a ternary phase consisting of carbon and manganese at temperature $T$ is expressed as

$$
\begin{aligned}
f^{\Theta}\left(c_{C}, c_{M n}, c_{F e}, T\right) & \equiv \frac{1}{V_{m}} G^{\Theta}\left(c_{C}, c_{M n}, c_{F e}, T\right) \\
& =\frac{1}{V_{m}}\left[A_{c}^{\Theta}(T) c_{C}^{2}+B_{c}^{\Theta}(T) c_{M n}^{2}+O_{c}^{\Theta}(T) c_{F e}^{2}\right. \\
& \left.+P_{c}^{\Theta}(T) c_{C}+Q_{c}^{\Theta}(T) c_{M n}+R_{c}^{\Theta}(T) c_{F e}+S_{c}^{\Theta}(T)\right],
\end{aligned}
$$

where the concentration of carbon, manganese and iron are treated in mole fractions, $c_{C}, c_{M n}$ and $c_{F e}$, respectively. Moreover, $A_{c}^{\Theta}(T), B_{c}^{\Theta}(T), O_{c}^{\Theta}(T), P_{c}^{\Theta}(T), Q_{c}^{\Theta}(T), R_{c}^{\Theta}(T)$ and $S_{c}^{\Theta}(T)$ are temperature- and phase-dependent coefficients which enable the incorporation of appropriate energy densities, and are ascertained around the equilibrium conditions.

The number of independent concentrations in the description of the energy density is conventionally reduced by imposing the summation constraint associated with mole fraction. Cor- 
respondingly, the concentration of the solvent-matrix is replaced through the relation

$$
c_{F e}=1-c_{C}-c_{M n},
$$

and the energy density is conventionally described based on carbon and manganese content. Although the introduction of the summation constraint in Eqn. (46) appears to be reasonable, it fails to differentiate components based on their lattice positions. In other words, reducing the number of independent concentration by imposing Eqn. (46) entails that all components occupy regular lattice and migrate substitutionally governed by diffusion potential. However, in ternary systems like manganese steel, the diffusion of one alloying element (carbon) is bound to interstitial sites and is dictated by chemical potential, while the other component (manganese) exhibits substitutional diffusion under the influence of diffusion potential. In order to differentiate the mode of diffusion, and the respective governing factor, free-energy density of the ternary is written as

$$
\begin{aligned}
G^{\Theta}\left(\rho_{C}, \rho_{M n}, \rho_{F e}, T\right) & =V_{m}^{2}\left[A^{\Theta}(T) \rho_{C}^{2}+B^{\Theta}(T) \rho_{M n}^{2}+O^{\Theta}(T) \rho_{F e}^{2}\right] \\
& +V_{m}\left[P^{\Theta}(T) \rho_{C}+Q^{\Theta}(T) \rho_{M n}+R^{\Theta}(T) \rho_{F e}\right]+S^{\Theta}(T),
\end{aligned}
$$

wherein the concentration of the components are treated in molar number-density. A numberdensity based formulation of free energy is rendered through Eqn. (17), and the coefficients associated with Eqn. (45) correspondingly vary in this description. Since the summation relation involving molar number-density, unlike Eqn. (46), is distinct for components occupying regular and interstitial sites, it can be employed to introduce the necessary disparity within the free-energy formulation. Therefore, based on the constraint relating the molar number-density of regular-lattice components, $\rho_{F e}+\rho_{M n}=\frac{1}{v_{\text {sub }}^{\ominus}}$, free energy of an individual phase replacing solvent-matrix concentration is written as

$$
\begin{aligned}
G^{\Theta}\left(\rho_{C}, \rho_{M n}, T\right) & =V_{m}^{2}\left\{A^{\Theta}(T) \rho_{C}^{2}+B^{\Theta}(T) \rho_{M n}^{2}+O^{\Theta}(T)\left[\frac{1}{v_{\text {sub }}^{\Theta}}-\rho_{M n}\right]^{2}\right\} \\
& +V_{m}\left\{P^{\Theta}(T) \rho_{C}+Q^{\Theta}(T) \rho_{M n}+R^{\Theta}(T)\left[\frac{1}{v_{\text {sub }}^{\Theta}}-\rho_{M n}\right]\right\}+S^{\Theta}(T),
\end{aligned}
$$

where $\frac{1}{v_{\text {sub }}^{\Theta}}$ is identical partial molar-volume of chemical species including solvent-matrix that are bound to regular lattice. Moreover, it is vital to realise that Eqn. (48) is analogous to Eqns. (29) 
and (43), wherein by exclusively introducing the summation constraint of regular-lattice species, the components are distinguished and their evolution are respectively coupled with diffusion or chemical potential depending on their lattice occupancy. In other words, for the Fe-C-Mn system considered in the present work, disparity between the alloying elements, and the factors governing their evolution, is established by Eqn. (48). Through straightforward algebraic operations, free energy formulation distinguishing components based on their lattice position can further be simplified as

$$
G^{\Theta}\left(\rho_{C}, \rho_{M n}, T\right)=\bar{A}^{\Theta}(T) \rho_{C}^{2}+\bar{B}^{\Theta}(T) \rho_{M n}^{2}+\bar{P}^{\Theta}(T) \rho_{C}+\bar{Q}^{\Theta}(T) \rho_{M n}+\bar{S}^{\Theta}(T)
$$

The phase- and temperature-dependent coefficients that dictate the free-energy density in above formulation reads

$$
\begin{aligned}
\bar{A}^{\Theta}(T) & =V_{m}^{2} A^{\Theta}(T) \\
\bar{B}^{\Theta}(T) & =V_{m}^{2}\left[B^{\Theta}(T)+O^{\Theta}(T)\right] \\
\bar{P}^{\Theta}(T) & =V_{m} P^{\Theta}(T) \\
\bar{Q}^{\Theta}(T) & =V_{m}\left[Q^{\Theta}(T)-R^{\Theta}(T)-2 \frac{V_{m}}{v_{\text {sub }}^{\Theta}} O^{\Theta}(T)\right] \\
\bar{S}^{\Theta}(T) & =\frac{V_{m}}{v_{\text {sub }}^{\Theta}}\left[\left(\frac{V_{m}}{v_{\text {sub }}^{\Theta}}\right) O^{\Theta}(T)+R^{\Theta}(T)+\left(\frac{v_{\text {sub }}^{\Theta}}{V_{m}}\right) S^{\Theta}(T)\right] .
\end{aligned}
$$

In systems with dilute carbon concentration, since molar and partial molar-volume can be approximated to be equal $\left(V_{m} \approx v_{\text {sub }}^{\Theta}\right)$, free-energy coefficients $\bar{Q}^{\Theta}(T)$ and $\bar{S}^{\Theta}(T)$ can be written as

$$
\begin{aligned}
\bar{Q}^{\Theta}(T) & =V_{m}\left[Q^{\Theta}(T)-R^{\Theta}(T)-2 O^{\Theta}(T)\right] \\
\bar{S}^{\Theta}(T) & =\left[O^{\Theta}(T)+R^{\Theta}(T)+S^{\Theta}(T)\right] .
\end{aligned}
$$

For the ternary alloy systems considered in the present analysis, the free-energy coefficients are estimated from TCFe8 CALPHAD database. Furthermore, the accuracy of the approximation is ensured by the ability of the free-energy formulation to recover equilibrium conditions. Thermodynamic parameters that primarily dictate the evolution of carbon and manganese, based on 
free energy approximation in Eqn. (49), is respectively expressed as

$$
\begin{array}{r}
\mu_{C}\left(\rho_{C}, T\right)=\frac{\partial f^{\Theta}\left(\rho_{C}, \rho_{M n}, T\right)}{\partial \rho_{C}}=2 \bar{A}^{\Theta}(T) \rho_{C}+P^{\Theta}(T) \\
\mu_{M n}\left(\rho_{M n}, T\right)=\frac{\partial f^{\Theta}\left(\rho_{C}, \rho_{M n}, T\right)}{\partial \rho_{M n}}=2 \bar{B}^{\Theta}(T) \rho_{C}+\bar{Q}^{\Theta}(T),
\end{array}
$$

where $\mu_{C}\left(\rho_{C}, T\right)$ and $\mu_{M n}\left(\rho_{M n}, T\right)$ correspond to the chemical and diffusion potential of carbon and manganese. Moreover, the predominant entries of the susceptibility matrix for the current Fe-C-Mn systems are

$$
\begin{gathered}
\chi_{C}^{\Theta}=\frac{\partial \rho_{C}}{\partial \mu_{C}}=\frac{1}{2 \bar{A}^{\Theta}(T)} \\
\chi_{M n}^{\Theta}=\frac{\partial \rho_{M n}}{\partial \mu_{M n}}=\frac{1}{2 \bar{B}^{\Theta}(T)},
\end{gathered}
$$

where $\chi_{C}^{\Theta}$ and $\chi_{M n}^{\Theta}$ are kinetic coefficients that respectively influence mobility of carbon and manganese during interstitial and substitutional diffusion. These entities of the susceptibility matrix are estimated from kinetics database MOBFe3 via DICTRA software package.

Entire approach of realising free-energy coefficients begins with the estimation of entries associated with susceptibility matrix, $\bar{A}^{\Theta}(T)$ and $\bar{B}^{\Theta}(T)$, from kinetic database. Subsequently, assuming that phase $-\alpha$ and $-\beta$ are in equilibrium, from Eqn. (52), the related parameters $P^{\Theta}(T)$ and $Q^{\Theta}(T)$ are determined. The constants $\bar{S}^{\Theta}(T)$ for $\Theta \in\{\alpha, \beta\}$ are finally ascertained from pre-determined coefficients. The approximation technique, delineated in this section, for incorporating CALPHAD data of manganese steel can directly be extended to any ternary system of the form Fe-C-X, wherein alloying element $(\mathrm{X})$ replaces solvent-matrix atoms in regular lattice with carbon occupying interstitial sites.

\section{Simulation set-up}

\subsection{Domain configuration}

In the present work, austenite to ferrite transformation in ternary Fe-C-Mn system is modelled by distinguishing the respective interstitial and substitutional diffusion of carbon and manganese through the re-formulated grand-potential based phase-field model. A quasi-one-dimensional set-up, wherein a noticeable width is rendered to otherwise one-dimensional domain, is adopted 

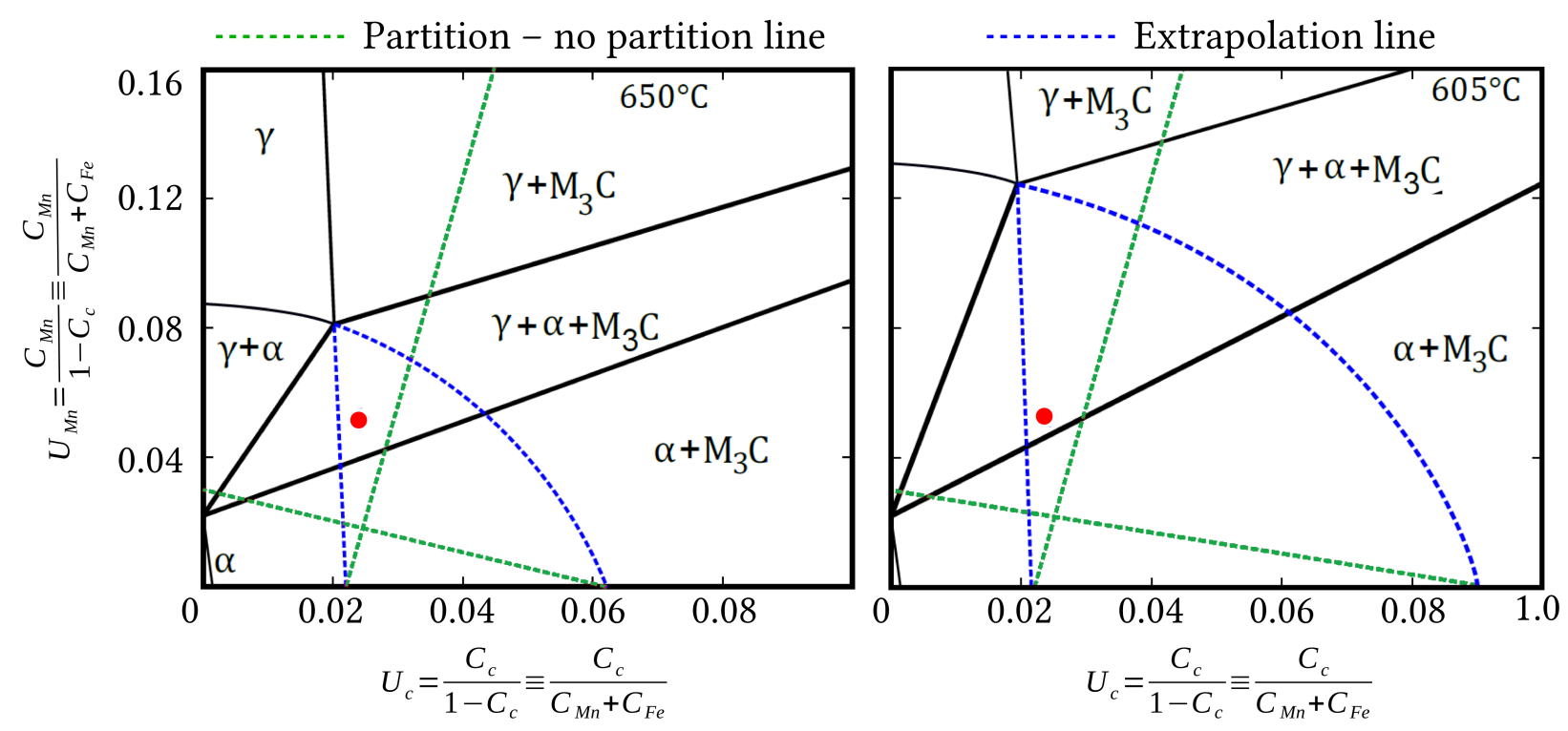

Figure 1: A section of isotherm pertaining to manganese steel at temperatures $650^{\circ} \mathrm{C}$ and $605^{0} \mathrm{C}$, wherein concentrations are expressed in U-fractions. The position of the alloy of composition 2.5 at.\% carbon and 5.4 at.\% manganese is shown in red solid dots.

for all simulations pertaining to austenite decomposition. This quasi-1D domain is of length $84 \mu \mathrm{m}$, while its width is $10 \mu \mathrm{m}$. The width of the quasi-1D domain ensures that the interface contribution including the effect of curvature is adequately encompassed, unlike conventional one-dimensional setup.

The simulation domain adopted in the current investigation is discretised through finitedifference scheme into identical grids with access to six adjacent cells in the principal directions. The equi-sized grids are of dimension $\Delta \mathrm{X}=\Delta \mathrm{Y}=0.04 \mu \mathrm{m}$. Equations pertaining to the temporal evolution of phase-field, chemical and diffusion potentials, Eqns. (33) and (34), are explicitly solved, in finite-difference framework, under forward marching Euler's scheme. No-flux Neumann boundary condition is imposed at the longitudinal ends of the quasi-1D domain, while laterally, along the width, periodic condition is assigned.

\subsection{Material parameters}

Isothermal evolution of ferrite in austenite-matrix of ternary manganese steel of composition 2.5at.\% carbon and 5.4at.\% manganese is modelled at temperatures $650^{\circ} \mathrm{C}$ and $605^{\circ} \mathrm{C}$ by 
Table 1: Coefficients describing free energies of ferrite and austenite at $650^{\circ} \mathrm{C}$ and $605^{0} \mathrm{C}$.

\begin{tabular}{|c||c|c|c|c|c|}
\hline $605^{0} \mathrm{C}$ & $\bar{A}^{\Theta}\left(\mathrm{Jmol}^{-1}\right)$ & $\bar{B}^{\Theta}\left(\mathrm{Jmol}^{-1}\right)$ & $\bar{P}^{\Theta}\left(\mathrm{Jmol}^{-1}\right)$ & $\bar{Q}^{\Theta}\left(\mathrm{Jmol}^{-1}\right)$ & $\bar{S}^{\Theta}\left(\mathrm{Jmol}^{-1}\right)$ \\
\hline \hline Ferrite & $23.8 \times 10^{-4}$ & $6.1 \times 10^{-5}$ & -0.15275 & -0.44934 & -33704.43 \\
\hline Austenite & $1.2 \times 10^{-4}$ & $1.6 \times 10^{-5}$ & -0.16156 & -0.46188 & -29317.08 \\
\hline \hline $650^{0} \mathrm{C}$ & & & & & \\
\hline \hline Ferrite & $9.1 \times 10^{-4}$ & $0.93 \times 10^{-5}$ & -0.14526 & -0.50723 & -36662.02 \\
\hline Austenite & $1.05 \times 10^{-4}$ & $0.26 \times 10^{-5}$ & -0.15688 & -0.51595 & -33179.99 \\
\hline
\end{tabular}

Table 2: Interdiffusivity matrix governing the evolution rate of chemical and diffusion potential.

\begin{tabular}{|c|cc||cc|}
\hline & $605^{0} \mathrm{C}$ & & $650^{0} \mathrm{C}$ & \\
\hline \hline $\boldsymbol{D}_{i k}^{\gamma}$ & $\mathrm{C}$ & $\mathrm{Mn}$ & $\mathrm{C}$ & $\mathrm{Mn}$ \\
\hline $\mathrm{C}$ & $1.31 \times 10^{-13}$ & $-1.47 \times 10^{-14}$ & $3.13 \times 10^{-11}$ & $-5.74 \times 10^{-14}$ \\
$\mathrm{Mn}$ & $1.56 \times 10^{-21}$ & $7.71 \times 10^{-21}$ & $-9.05 \times 10^{-19}$ & $1.34 \times 10^{-18}$ \\
\hline \hline $\boldsymbol{D}_{i k}^{\alpha}$ & $\mathrm{C}$ & $\mathrm{Mn}$ & $\mathrm{C}$ & $\mathrm{Mn}$ \\
\hline $\mathrm{C}$ & $2.56 \times 10^{-11}$ & $-4.63 \times 10^{-12}$ & $4.81 \times 10^{-11}$ & $-1.04 \times 10^{-16}$ \\
$\mathrm{Mn}$ & $-4.34 \times 10^{-19}$ & $1.17 \times 10^{-18}$ & $-1.71 \times 10^{-18}$ & $1.54 \times 10^{-17}$ \\
\hline
\end{tabular}

employing the present re-formulated technique. The position of this composition in isotherms associated with the appropriate temperature is shown in Fig. 1. As illustrated in Fig. 1, the ternary manganese steel considered for the current analysis pertains to the three-phase regime, at both temperatures. Consequently, the phase transformation ultimately yields a combination of ferrite and austenite in definite phase-fraction. Since the equilibrium volume fraction of the phases, at a given temperature, for an alloy of definite composition is quantitatively predicted by CALPHAD database, the outcomes of the re-formulated approach are verified by comparing the phase-fractions. Moreover, as shown in Fig. 1. the ternary manganese steel, at temperatures $650^{\circ} \mathrm{C}$ and $605^{\circ} \mathrm{C}$, lies above the 'partitioning- and negligible-partitioning local-equilibrium' (PLE and NP-LE) transition line, thus implying that the phase transformation is accompanied by the diffusion of both carbon and manganese.

Quantitative driving-force for the growth of ferrite, as delineated in previous section, is incor- 


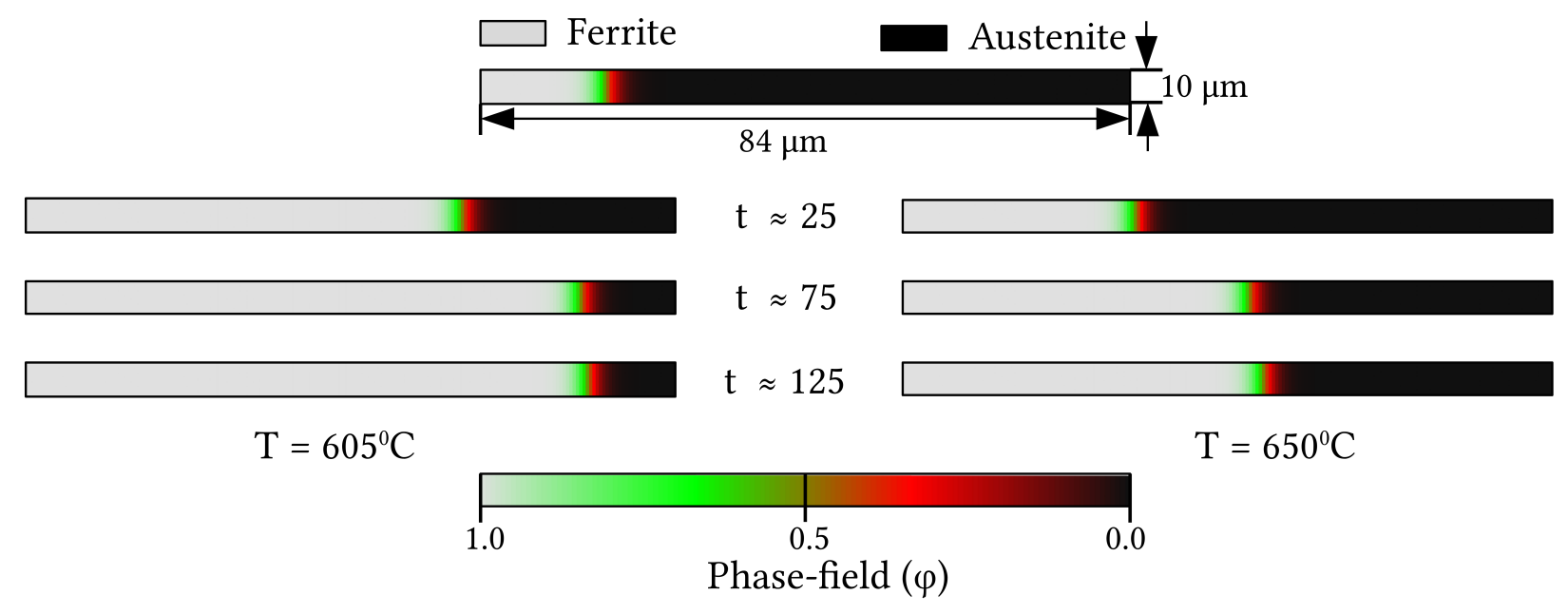

Figure 2: Temporal evolution of ferrite in austenite matrix at temperatures $650^{\circ} \mathrm{C}$ and $605^{0} \mathrm{C}$ in a quasi onedimensional simulation domain, wherein $t$ denotes the non-dimensionalised time.

porated through the polynomial approximation of energy densities rendered by TCFe8 database. The phase- and temperature-dependent coefficients which enable the introduction of appropriate bulk contribution is listed in Table. 1. Furthermore, the entries of the interdiffusivity matrix obtained from MOBFe3 database is presented in Table. 2 Kinetics parameters associated with the second-derivative of free-energy can be ascertained from $\bar{A}^{\Theta}$ and $\bar{B}^{\Theta}$, and incorporated in susceptibility matrix. Given the concentration of carbon in the manganese steel, the alloy is reasonably treated as dilute. Correspondingly, molar and partial molar-volume are considered to be identical, and of value $V_{m}=v_{\text {sub }}=7.17 \times 10^{-6} \mathrm{~m}^{-3} \mathrm{~mol}^{-1}$. Furthermore, an interfacial energy density of $\gamma=0.49 \mu \mathrm{m}^{-2}$ is assigned [43, 44, 45]. The width of the diffuse interface is fixed through length-scale parameter $\varepsilon=3 \times \Delta \mathrm{X}$. Properties of the interface pertaining to the present formalism, including the width and corresponding phase-field profile in the diffuse region, is briefly discussed in Appendix. The stability of the interface during the temporal evolution of phase-field is ensured by the relaxation parameter, which is set at $\tau=1.72 \times 10^{9} \mathrm{Jsm}^{-4}$. 


\section{Result and discussion}

\subsection{Phase transformation in quasi-1D domain}

The ternary manganese steel is allowed to evolve towards the phenomenological minimisation of its energy-densities, after introducing the appropriate bulk contribution and material parameters. The isothermal growth of ferrite in quasi-1D domain at temperatures $650^{\circ} \mathrm{C}$ and $605^{0} \mathrm{C}$, under the current formulation, is illustrated in Fig. 2 by considering the spatio-temporal change in phase-field $(\phi)$. The time $t$ included in this, and all subsequent, illustration is nondimensionalised by $\frac{V_{m} \kappa T}{D \gamma L^{3}}$, where $L$ is the diffusion length of carbon which is assumed to be $0.02 \mathrm{~m}[46]$.

At both temperatures, $650^{\circ} \mathrm{C}$ and $605^{\circ} \mathrm{C}$, ferrite progressively grows in austenite as shown in Fig. 2. However, it is evident from the illustration the transformation rate is noticeably faster at $605^{0} \mathrm{C}$, when compared to $650^{\circ} \mathrm{C}$. For instance, when viewed in relation to $650^{\circ} \mathrm{C}$, at $t=25$, the volume (area) fraction of ferrite is noticeably higher for large undercooling $\left(605^{0} \mathrm{C}\right)$. Furthermore, while there is small degree of phase transformation between $t=75$ to $t=125$ at $650^{\circ} \mathrm{C}$, the representative microstructure at $605^{0} \mathrm{C}$ seemingly reaches equilibrium phase-fraction at $t=75$, and remains unchanged subsequently.

In order to quantitatively evaluate the phase transformations rendered by the re-formulated approach, the increase in the volume fraction of ferrite with time is monitored and plotted in Fig. 3. Moreover, the equilibrium phase-fraction predicted by CALPHAD database is included in the illustration. In corroboration with the quasi-1D microstructural evolution in Fig. 2 the change in the volume-fraction of ferrite presented in Fig. 3 indicates that the transformation is rather sluggish at high temperature when compared $605^{0} \mathrm{C}$. However, despite the considerable disparity in the transformation rate, the progressive evolution of ferrite at both temperatures establish equilibrium phase-fraction as predicted by the TCFe8 database. This agreement between the equilibrium volume-fraction of the phases rendered by the simulations and the CALPHADinformed prediction indicates that re-formulated grand-potential model, through the incorporation of appropriate driving forces, is capable of yielding quantitative results. Since the primarily motive of the present analysis is to explicate the competence of the extended approach to distin- 


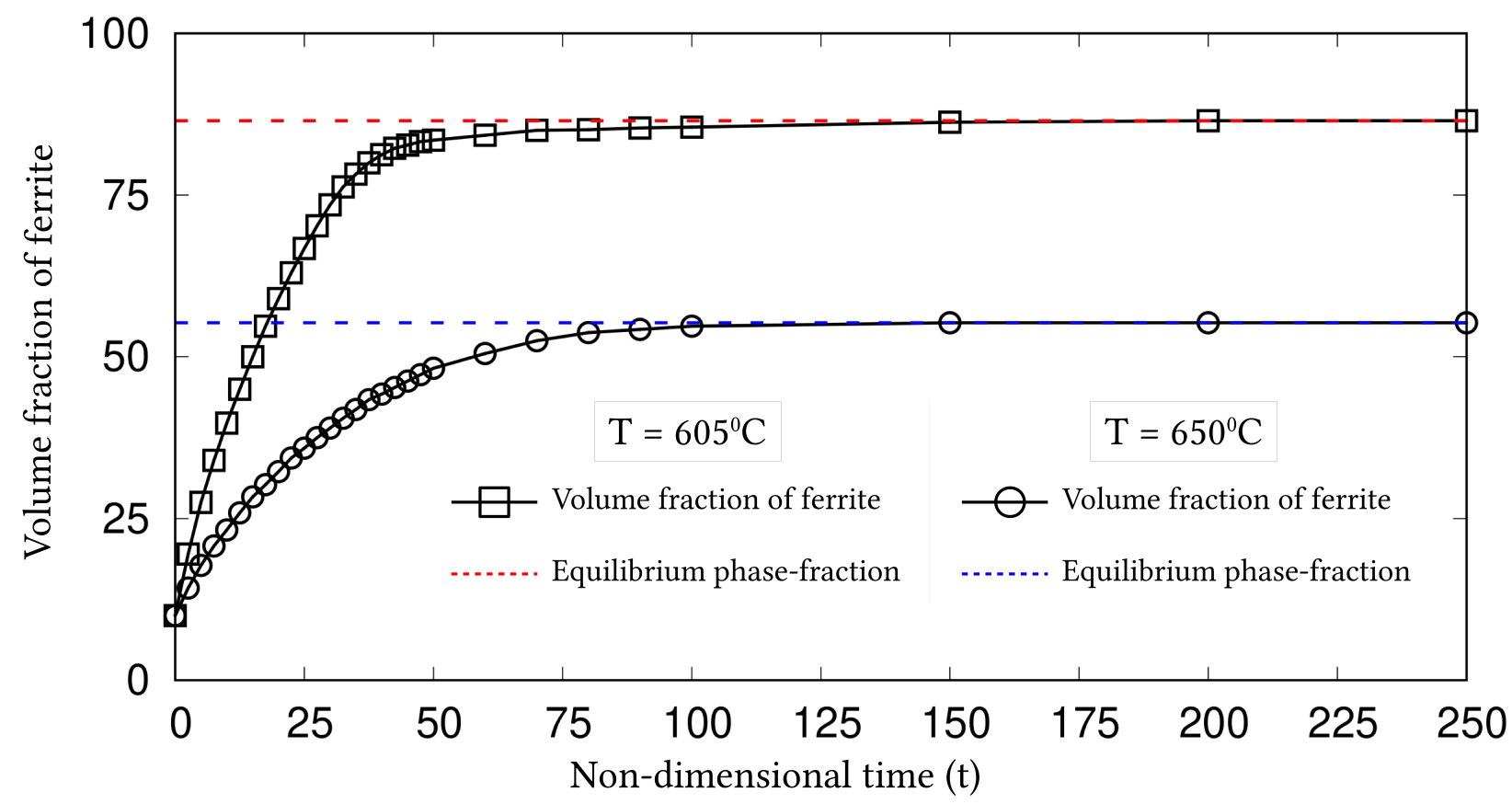

Figure 3: Progressive change in the volume fraction of ferrite which accompanies austenite decomposition at temperatures $650^{\circ} \mathrm{C}$ and $605^{\circ} \mathrm{C}$. The equilibrium phase-fraction for different undercoolings is estimated from CALPHAD database, and included. 


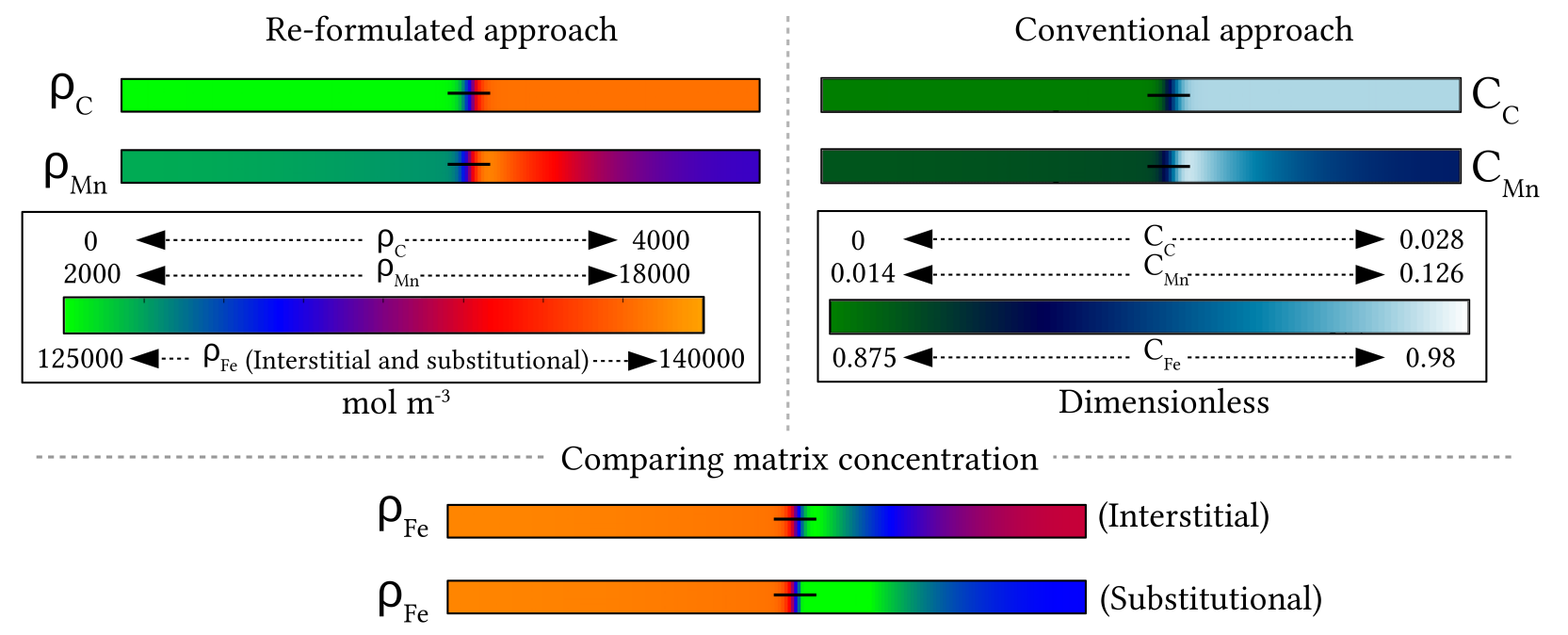

Figure 4: Concentration distribution of alloying elements, carbon and manganese, at $t=15$ of larger undercooling phase transformation $\left(605^{0} \mathrm{C}\right)$ resulting from conventional ('Substitutional') and current re-formulated technique ('Interstitial'). The matrix-distribution (Fe) from the mole-fraction based approach is converted to number-density for comparison with the corresponding outcome of the present model.

guish interstitial and substitutional diffusion, investigations asserting its consistency is restricted to this section (Fig. 3).

\subsection{Difference in concentration distribution}

During a phase transformation, concentration distribution of components, particularly, solventmatrix $(\mathrm{Fe})$, reflects the mechanism of diffusion. In other words, depending on the mode of mass transfer adopted by the alloying elements, substitutional or interstitial, the distribution of the solvent-matrix varies. While an exclusive interstitial diffusion leaves the matrix atoms unperturbed, a characteristic coupling between solvent and migrating element is observed during substitutional diffusion. In ternary Fe-C-Mn system, owing to the strictly interstitial diffusion of carbon, the concentration distribution of iron is apparently not disturbed by its migration. On the contrary, in a rather inaccurate framework, wherein carbon is treated as substitutionally migrating species, its diffusion is inherently coupled with the distribution of solvent-matrix. Therefore, the ability of an approach to distinguish interstitial and substitutional diffusion can be perceived from the concentration distribution it yields. Correspondingly, the concentration 
distribution rendered by the conventional, and the re-formulated phase-field technique for the transformation pertaining to $605^{0} \mathrm{C}$ at $t=15$ is shown in Fig. 4.

In Fig. 4. the concentration distribution of the alloying elements are appropriately represented in based on the formalism. While number-density is adopted to represented the outcomes of the re-formulated approach, the carbon and manganese distributions of conventional technique are illustrated based on mole-fraction. However, the distribution of matrix $(\mathrm{Fe})$ rendered by the regular treatment is converted to number-density, through Eqn. (17), for comparison with the results of the present formulation.

Owing to the equivalent bulk-contributions, and other kinetic parameters, both conventional and re-formulated grand-potential treatments visibly similar distribution of alloying elements, manganese and carbon. On the other hand, the regular and present approaches yield different spatial distribution of solvent-matrix concentration. The noticeable disparity in the distribution of iron is primarily due to the coupling of its spatial concentration with carbon diffusion. In other words, the conventional technique by treating the chemical composition in mole fraction, through its summation constraint, couples the evolution of all alloying elements with the solvent-matrix atoms. Accordingly, the migration of the chemical species reflect substitutional diffusion. By expressing the concentration in molar number-density, and distinguishing them through appropriate relation, in contrast, the present approach decouples the diffusion of carbon and iron in the Fe-C-Mn system as shown in Fig. 4. Despite the decoupling of carbon and iron diffusion, the migration of manganese is consistently related to solvent-matrix atoms by $\rho_{F e}+\rho_{M n}=\frac{1}{v_{\mathrm{sub}}^{\Theta}}$. This concept of treating the concentration in molar number-density ultimately renders a phase transformation governed by respective interstitial and substitutional diffusion of carbon and manganese.

An one-dimensional profile of the concentration of iron, carbon and manganese across the diffuse interface is shown in Fig. 5. For comparative analysis, all concentration profiles of the mole-fraction based model is converted to number density and included in the illustration. Owing to the equivalence of the driving-force, and identical supersaturation, the profile of the alloying elements emerging from the different treatments coincide with each other. Therefore, in Fig. 5 single concentration profile is adopted for each alloying element, despite the difference in the 


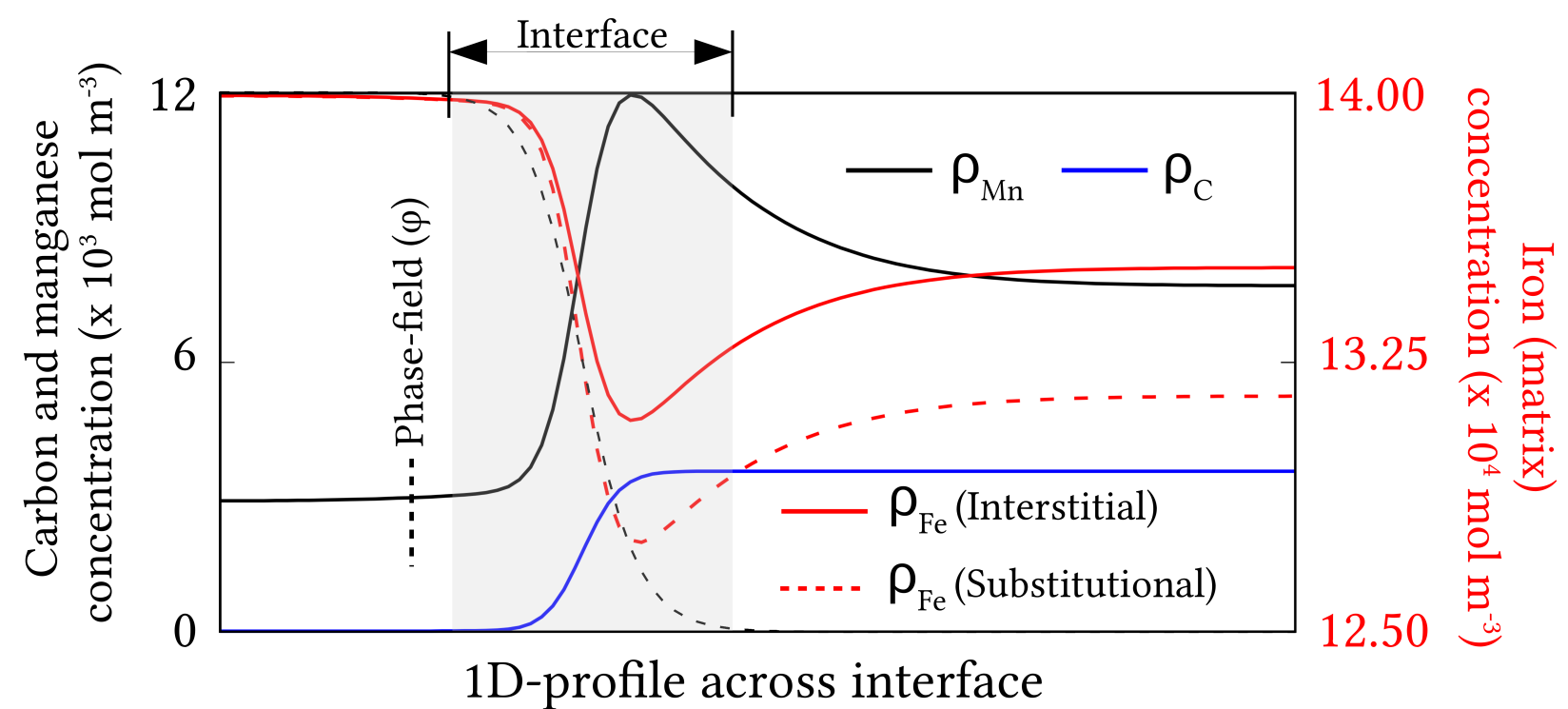

Figure 5: A one-dimensional concentration profile of alloying elements, and solvent-matrix across the diffuse interface of the conventional and re-formulated grand-potential model.

approaches.

As opposed to the alloying elements, Fig. 5 unravels that the profile of iron concentration resulting from the current technique is significantly different from the ones rendered by the conventional model. This disparity is the reflection of the dissimilarity in the distribution of solventmatrix concentration illustrated in Fig. 4. In other words, the difference in the 1D-concentration profile is due to the inaccurate coupling of carbon and solvent-matrix composition in regular grand-potential model. This ambiguity can be rectified, and a profile akin to one rendered by present approach can be recovered by decoupling iron and carbon concentration. Furthermore, Fig. 5 indicates that there exists a significant gradient in the distribution of manganese in austenite, when compared to carbon. This difference in the concentration profile amongst the alloying elements is principally due to the overall composition of the system. As shown in Fig. 1, the alloy composition pertains to the partition regime of the ternary isotherm, thereby the phase transformation is primarily governed by the diffusion of manganese. And moreover, the kinetic coefficients dictating the migration of carbon is noticeably higher than that of manganese as indicated in Table.2 Therefore, while the spatial concentration of carbon in austenite is seemingly 
Table 3: Free energy coefficients pertaining to ferrite and austenite of 4.8 at. $\%$ carbon and 0.5 at. $\%$ manganese alloy at $605^{0} \mathrm{C}$.

\begin{tabular}{|c||c|c|c|c|c|}
\hline $605^{0} \mathrm{C}$ & $\bar{A}^{\Theta}\left(\mathrm{Jmol}^{-1}\right)$ & $\bar{B}^{\Theta}\left(\mathrm{Jmol}^{-1}\right)$ & $\bar{P}^{\Theta}\left(\mathrm{Jmol}^{-1}\right)$ & $\bar{Q}^{\Theta}\left(\mathrm{Jmol}^{-1}\right)$ & $\bar{S}^{\Theta}\left(\mathrm{Jmol}^{-1}\right)$ \\
\hline \hline Ferrite & $12.5 \times 10^{-4}$ & $2.4 \times 10^{-5}$ & -0.64192 & -0.23080 & -38614.47 \\
\hline Austenite & $3.4 \times 10^{-4}$ & $2.4 \times 10^{-5}$ & -0.65928 & -0.23080 & -22577.31 \\
\hline
\end{tabular}

uniform, a gradient is observed in the distribution of manganese.

\subsection{Phase transformation under para-equilibrium}

Generally, during a reconstructive transformation, all the associated components diffuse to render characteristic composition of the phases. Moreover, the concentrations are considered to reflect equilibrium composition of the corresponding phases at the interface. Such transformations which are characterised by diffusion of all alloying elements and local-equilibrium (LE) at the interface separating the evolving and parent phase are said to occur under 'orthoequilibrium'. However, in some alloys including manganese steels, phase-changes under definite conditions are exclusively governed by diffusion of one (or few) alloying element(s). Concentration of remaining alloying elements, in addition to solvent-matrix, remains undisturbed despite migration of specific component(s). In Fe-C-X system, these 'para-equilibrium' governed phase-transformations often involve interstitial diffusion of carbon alone. Owing to the inherent coupling of the composition of alloying elements with the solvent-matrix, the conventional grand-potential approach cannot be adopted to model phase-changes related to paraequilibrium. By sufficiently distinguishing interstitial diffusion from the substitutional migration of atoms, the re-formulated technique facilitates the modelling of para-equilibrium governed phase-transformation.

The ability of the current formulation to model austenite to ferrite transformation under para-equilibrium is elucidated by considering an alloy of composition 4.8 at.\% carbon and 0.5 at.\% manganese. Even though this composition pertains to the negligible-partitioning localequilibrium (NP-LE) section of the $605^{\circ} \mathrm{C}$ isotherm, para-equilibrium is reasonably assumed, considering that both conditions exclusively involve carbon diffusion, and disparity is related 

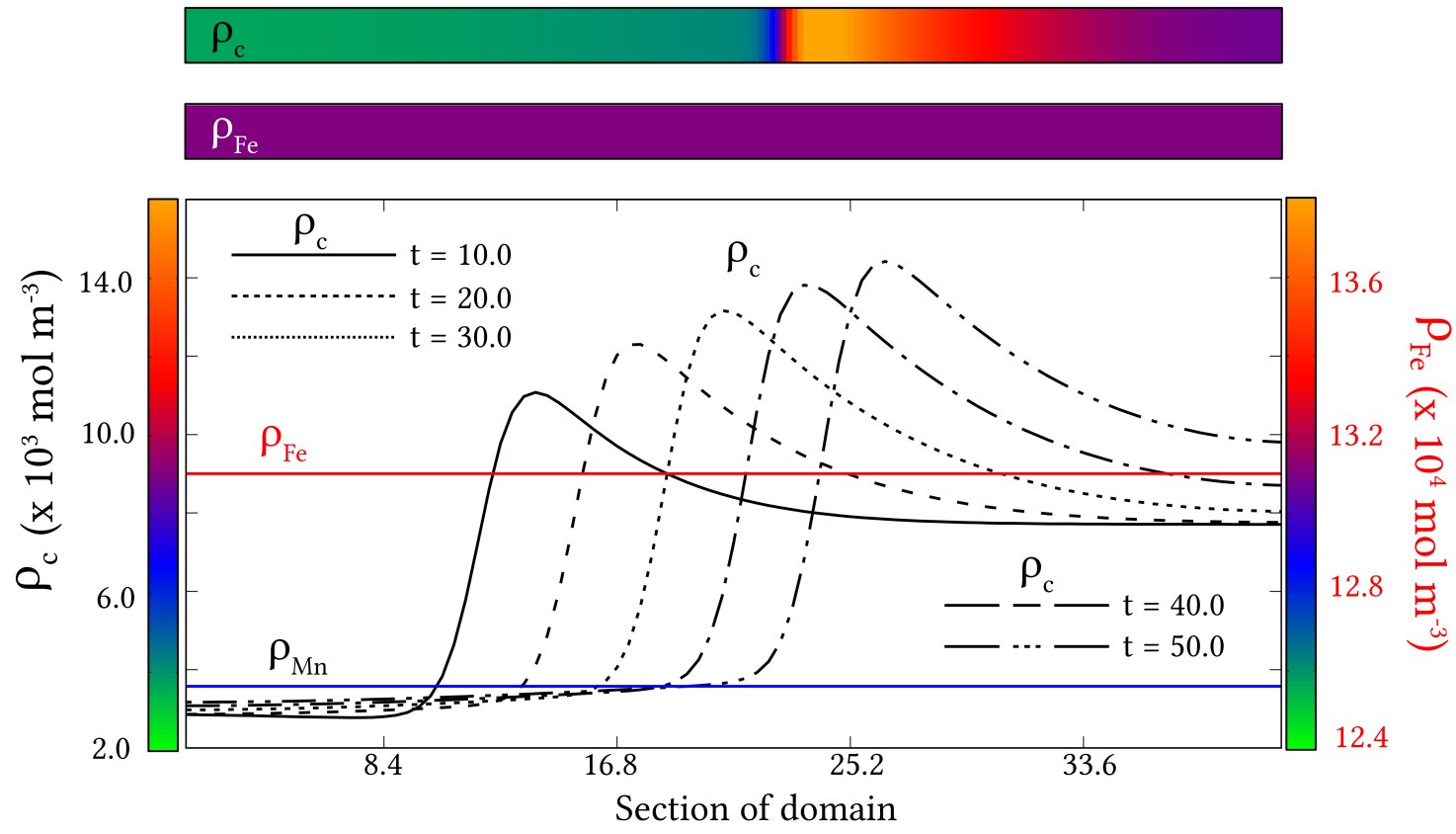

Figure 6: One-dimensional representation of concentration evolution, including carbon, manganese and iron, during the growth of ferrite under para-equilibrium. The corresponding distribution of carbon and solvent-matrix in quasi1D setup resulting from the re-formulated approach is appended.

to the accumulation of manganese at the interface of NP-LE governed transformation. The freeenergy coefficients which enable the incorporation of appropriate driving-force is listed in Table. 3 Moreover, by assigning identical coefficients, which dictate chemical potential of manganese, to both phases, it is ensured that Mn remains unperturbed all through the evolution.

Instead of illustrating the progressive growth of ferrite through spatio-temporal change in phase-field, the corresponding evolution of concentration pertaining to the alloying elements and solvent-matrix is considered. For this depiction, a section of quasi-1D domain which encompasses the diffuse interface is appropriated. The one-dimensional concentration profile of carbon, manganese and iron, and their temporal change accompanying the phase transformation, as rendered by the re-formulated model is shown in Fig. 6 Consistent with the description of paraequilibrium, the present technique yields a transformation wherein distribution of manganese and iron content remain unaltered all through evolution, while the phases evolve exclusively governed by carbon diffusion. The change in the equilibrium profile of carbon with time reflects 

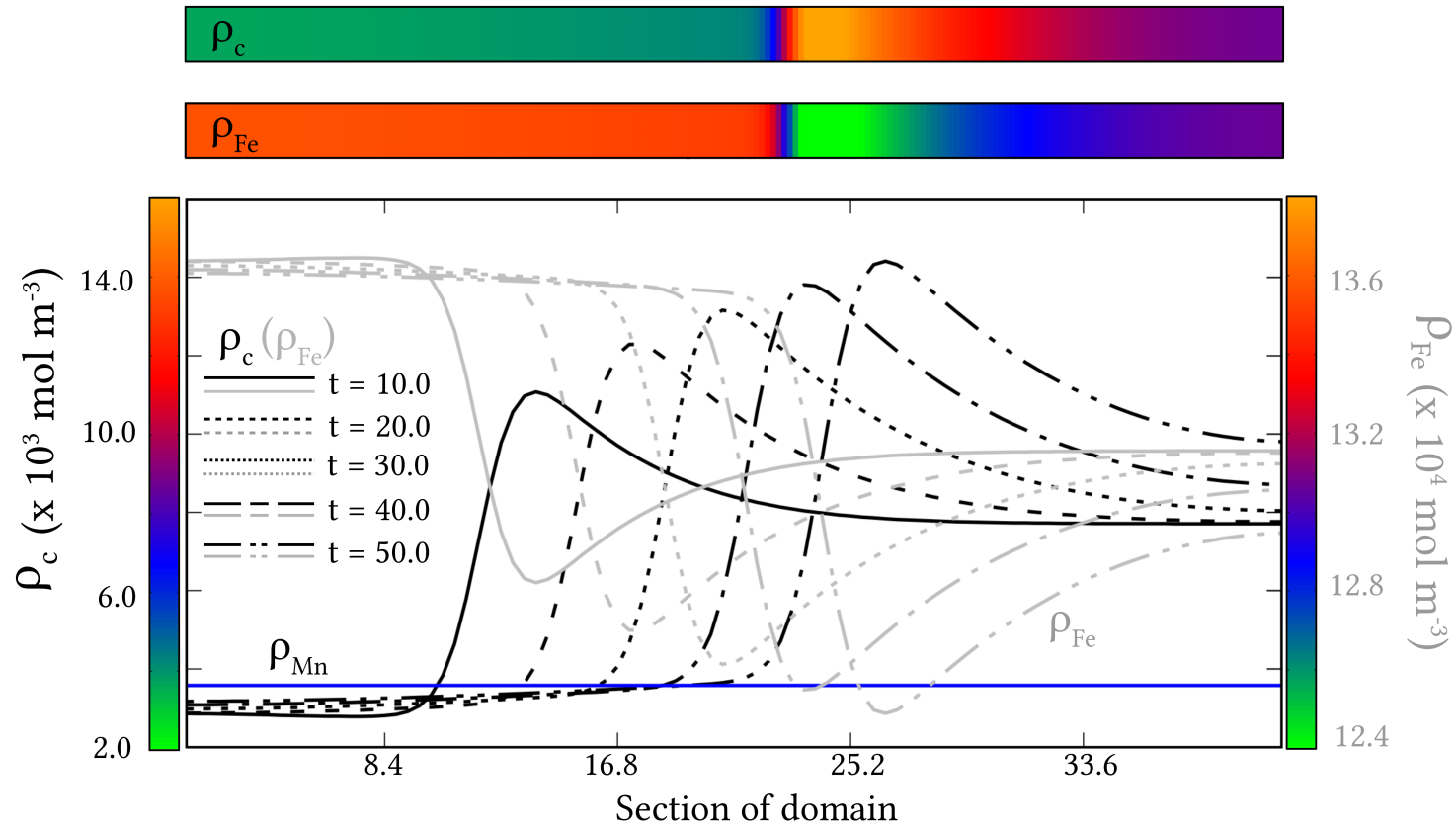

Figure 7: Concentration distribution and its evolution rendered by conventional grand-potential based model under identical conditions associated with Fig. 6

the non-steady state equilibrium condition that exists at the interface, which is extensively discussed elsewhere [47, 48]. Moreover, such apparent increase in the carbon-concentration peak corroborates with previous numerical simulations wherein molar number-density is adopted to distinguish interstitial and substitutional diffusion in Fe-C-Mn system [26].

Analogous evolution of phases is simulated by adopting conventional model, and incorporating equivalent driving-force. The corresponding temporal change in concentration profiles of alloying elements, and solvent-matrix iron is shown in Fig. 7. Although the conventional technique seemingly yields identical profile for carbon, its coupling with the solvent-matrix correspondingly alters its concentration profile, thereby introducing a rather non-physical spatial disparity in the composition of iron. Furthermore, the inadequacy of the existing technique to simulate phase transformation under para-equilibrium is unraveled by the apparent non-uniform distribution of iron, as illustrated in the quasi-1D representation of Fig. 7 
Table 4: Free energy coefficients which facilitate almost complete expulsion of carbon from martensite of 4.8 at. $\%$ carbon and 0.5 at. $\%$ manganese alloy at $605^{\circ} \mathrm{C}$.

\begin{tabular}{|c||c|c|c|c|c|}
\hline $605^{0} \mathrm{C}$ & $\bar{A}^{\Theta}\left(\mathrm{Jmol}^{-1}\right)$ & $\bar{B}^{\Theta}\left(\mathrm{Jmol}^{-1}\right)$ & $\bar{P}^{\Theta}\left(\mathrm{Jmol}^{-1}\right)$ & $\bar{Q}^{\Theta}\left(\mathrm{Jmol}^{-1}\right)$ & $\bar{S}^{\Theta}\left(\mathrm{Jmol}^{-1}\right)$ \\
\hline \hline Martensite & $31.3 \times 10^{-4}$ & $2.4 \times 10^{-5}$ & -0.36324 & -0.23080 & -25667.2 \\
\hline Austenite & $1.65 \times 10^{-4}$ & $2.4 \times 10^{-5}$ & -0.43819 & -0.23080 & -22577.31 \\
\hline
\end{tabular}

\subsection{Carbon partitioning under constrained-carbon-equilibrium}

Combination of desired properties like strength and toughness is achieved in steels by an unique heat treatment process called quenching and partitioning [49]. This technique involves quenching an austenised steel at a particular temperature to establish a pre-determined phasefraction of martensite and austenite. Subsequently, the stability of austenite at low temperatures is enhanced by facilitating the diffusion of carbon from the martensite to the surrounding matrixphase. Microstructure emerging from this heat treatment process comprises of martensite and austenite, which offers an excellent combination of mechanical properties.

The diffusion of carbon from martensite to austenite during partitioning is characterised by a negligible shift in the interface position, and generally unchanging concentration of other alloying elements, particularly one(s) occupying the regular lattice [50]. Therefore, this carbon partitioning is ascribed to a unique equilibrium condition called constrained-carbon-equilibrium. The characteristic diffusion of carbon which accompanies quenching and partitioning heat treatment is modelled using the present re-formulated approach. Conventional models that treat concentration in mole fractions have hitherto been employed to model the carbon partitioning [51, 52, 53]. Although such simulations apparently yield a distribution of carbon consistent with the imposed difference in the chemical potential, the ensuing change in the distribution of solvent-matrix iron is largely overlooked. The inaccuracy associated with conventional treatments is rectified by the outcomes of current model.

Volume fraction of phases during carbon diffusion associated with quenching and partitioning is significantly different from its equilibrium phase-fraction. Therefore, the endpoints of partitioning are considered to be different from equilibrium composition, and are specifi- 

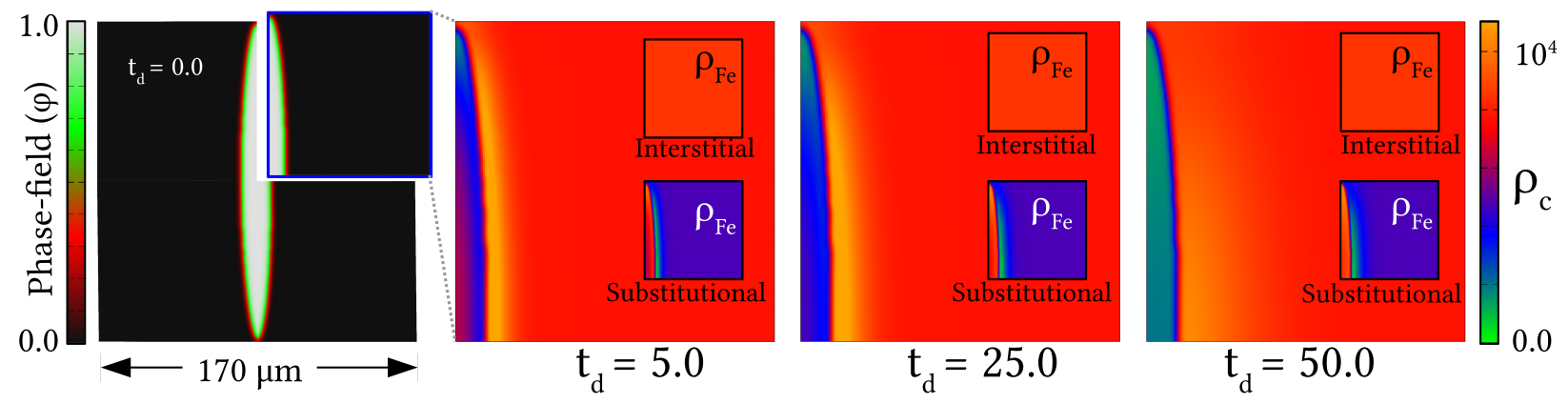

Figure 8: Partitioning of carbon from a quadrant of the martensite sheave. Corresponding change in the distribution of iron rendered by conventional and re-formulated grand-potential approach is included in sub-plots.

cally ascertained based on phase-fraction. Considerable amount of carbon is generally expelled out of martensite during partitioning. Accordingly, free-energy coefficients that ensure almost complete expulsion of carbon from martensite is involved in present simulation. These coefficients are listed in Table. 4, and an overall alloy composition identical to previous section is adopted for modelling partitioning under constrained-carbon-equilibrium. Furthermore, akin to para-equilibrium, the diffusion of manganese is averted by establishing a corresponding partialequilibrium between the phases.

A simulation domain comprising of a martensite sheave, as shown in Fig. 8 is devised for present analysis. The phases are differentiated by assigning appropriate values to the phasefields. Adhering to the description of constrained-carbon-equilibrium, the spatio-temporal changes in the phase-fields are not considered for the current simulation. However, change in concentration distribution with time is exclusively modelled by solving the evolution of the corresponding chemical potential. The simulation domain is initialised by a uniform composition pertaining to 4.8 at.\% carbon and 0.5 at.\% manganese, and the carbon is allowed to diffuse governed by the difference in the chemical potential. Owing to the symmetry of the domain, the progressive change in the distribution of carbon concentration is illustrated by considering a quadrant.

The competence of the re-formulated approach to distinguish interstitial and substitutional diffusion is noticeably unraveled in Fig. 8. Present technique ensures that the distribution of iron remains homogeneous and unperturbed, despite the partitioning of carbon. However, on the other hand, a spatial inhomogeneity in solvent-matrix concentration complementing the carbon 
distribution is inherently introduced by the conventional model, as shown in the subplots of Fig. 8 Eventhough, both techniques convincingly capture the principal aspect of partitioning, which is the temporal change in carbon distribution, only the current approach is consistent with the thermodynamic description, and thus, can be adopted further analysis wherein the role of elasticity and other influencing factors are considered.

\section{Conclusion}

Theoretical techniques, particularly phase-field models, are often formulated in multicomponent framework to encompass highly applicable materials that generally comprise of several alloying elements. However, owing to the rather conventional treatment of concentration, these techniques are restricted to materials wherein atoms of the alloying elements replace the solventmatrix in regular lattice-sites. Therefore, microstructural evolutions in wide range of alloys, for instance steels, cannot be principally modelled through the regular phase-field techniques, considering that one or more alloying elements exclusively occupy interstitial lattice, and their migration is rigidly confined to their sites. Additionally, adopting mole fraction to describe composition of a multicomponent system is inherently inadequate for imposing definite equilibrium conditions including para-equilibrium. In the present work, the aforementioned limitations of existing phase-field models are addressed by re-formulating grand-potential based technique which is generally posed as an efficient approach for handling multicomponent systems.

The ability of the re-formulated technique to accurately grasp phase transformation in multicomponent systems with interstitial alloying-elements is explicated by modelling austenite decomposition in manganese steel (Fe-C-Mn). The framework of the current which lends itself to impose unique equilibrium condition is demonstrated by simulating growth of ferrite under para-equilibrium. Moreover, the exclusive migration of carbon, which associated with quenching and partitioning heat treatment technique, governed by constrained-para-equilibrium is convincingly modelled through the reformulated grand-potential model. In addition to the phase-field approach, the approximation technique delineated in this work facilitates the incorporation of CALPHAD data of multicomponent systems with regular and interstitial alloying elements. 
Present approach distinguishes interstitial and substitutional diffusion of components based on Eqn. (15), wherein identical partial molar-volume is assumed for species occupying regular lattice, while influence of interstitial elements on volume is deemed negligible. Despite its criticality, this assumption negates the elastic contribution primarily rendered by the interstitial and also regular-lattice components in phase transformations. Realising this limitation of the re-formulated technique, subsequent works would adopt existing chemo-elastic framework to encompass the elastic contribution of the alloying elements [53, 33].

Additionally, given that this work is a preliminary attempt to extend the applicability of grand-potential based models to wide range of materials with interstitial components, the formulation is restricted to two-phase systems. Therefore, in the subsequent investigations, the re-formulated technique would be delineated in multiphase-field framework. Moreover, since the current analysis is primarily focused on exhibiting the ability of the phase-field approach to distinguish interstitial and substitutional diffusion, rather straightforward representative microstructural evolutions are considered. In the upcoming works, the re-formulated grand-potential model will be adopted to investigate convoluted phase transformation like growth of divergent pearlite, and austenite decomposition under Negligible-Partitioning Local-Equilibrium condition (NPLE). The existing framework of the present technique will be extended in near future to encompass elastic and plastic driving forces.

\section{Appendix: Interface properties}

The properties of the diffuse interface emerging from the present formalism is discussed by considering a one-dimensional system.

The overall grand-potential based energy-density of the 1D system, is expressed as

$$
\Omega\left(\boldsymbol{\mu}, \phi, \boldsymbol{\nabla}_{x} \phi\right)=\int_{V} \mathrm{~d} V\left[\psi\left(\boldsymbol{\rho}^{\alpha}(\boldsymbol{\mu}), \boldsymbol{\rho}^{\beta}(\boldsymbol{\mu}), \phi\right)+\frac{1}{\varepsilon} f_{\text {pen }}(\phi)+\varepsilon \gamma\left|\boldsymbol{\nabla}_{x} \phi\right|^{2}\right] . \quad \text { (Appendix .1) }
$$

In order to realise the interface properties, an equilibrium is established in the system through appropriate composition, $\boldsymbol{\rho}_{\mathrm{eq}}^{\alpha}$ and $\boldsymbol{\rho}_{\mathrm{eq}}^{\beta}$. This equilibrium through the absolute negation of the bulk driving-force, $\Delta \psi(\boldsymbol{\mu}) \equiv \psi_{\alpha}(\boldsymbol{\mu})-\psi_{\beta}(\boldsymbol{\mu})=0$, creates a static system that is devoid any interface 
migration, and characterised by

$$
\tau \varepsilon \frac{\partial \phi(x, t)}{\partial t}=-\frac{\delta \Omega\left(\boldsymbol{\mu}, \phi, \boldsymbol{\nabla}_{x} \phi\right)}{\delta \phi}=0
$$

(Appendix .2)

The time-independent scalar variable typifying the one-dimensional static system is represented by $\phi_{0}(x)$.

Owing to the lack of bulk contribution, the evolution Eqn. Appendix .2 yields the relation

$$
2 \gamma \varepsilon \frac{\mathrm{d}^{2} \phi_{0}(x)}{\mathrm{d} x^{2}}=\frac{\gamma}{\varepsilon} \frac{16}{\pi^{2}}\left(\frac{\mathrm{d}}{\mathrm{d} \phi_{0}}\left[\phi_{0}^{2}\left(1-\phi_{0}\right)^{2}\right]\right)
$$

Following Refs. [19, 20], the equilibrium profile of the static phase-field is ascertained by integrating the above expression. For the penalising potential of the form $f_{\text {pen }}(\phi)=\frac{16}{\pi^{2}} \phi^{2}(1-\phi)^{2}$, the spatial profile of the phase-field at the diffuse interface is expressed as

$$
\phi_{0}(x)=\frac{1}{2}\left[1-\tan ^{-1}\left(\frac{2}{\pi \sqrt{\varepsilon}} x\right)\right] .
$$

From the interface profile delineated in the above Eqn. (Appendix .4), the width of the diffuse region can be determined by $\int_{0}^{\Lambda} \mathrm{d} x$, and expressed as

$$
\Lambda=\tilde{a}_{0} \frac{\pi \varepsilon}{2},
$$

where $\tilde{a}_{0}$ is a constant which varies with the limits of the phase-field employed for calculating interface width. In other words, the current form of the penalising double-well potential does not permit the limits $\phi=0$ and $\phi=1$ for the calculation of the interface width. Therefore, limits like 0.1 and 0.9 or 0.05 and 0.95 are generally chosen, and the corresponding constant $\tilde{a}_{0}=2.2$ or 2.94 is involved [19, 20].

\section{Declaration of interest}

The authors declare that there are no known competing interests .

\section{Acknowledgments}

PGK Amos thank the financial support of the German Research Foundation (DFG) under the project AN 1245/1. The combined model provides a fundamental technique that can be applied for 
further research within the 'VirtMat - Virtual Material Design' initiative funded by the Helmholtz association which is gratefully acknowledged by Britta Nestler. The authors thank Prof. Leslie Mushongera, Dr. Daniel Schneider, and Ephraim Schoof for the fruitful discussion and support.

\section{References}

\section{References}

[1] Ahmad K Elshennawy and Gamal S Weheba. Manufacturing processes \& materials. Society of Manufacturing Engineers (SME), 2015.

[2] Koenraad George Frans Janssens, Dierk Raabe, Ernest Kozeschnik, Mark A Miodownik, and Britta Nestler. Computational materials engineering: an introduction to microstructure evolution. Academic Press, 2010.

[3] Nikolas Provatas and Ken Elder. Phase-field methods in materials science and engineering. John Wiley \& Sons, 2011.

[4] John M Chadam and Helen Rasmussen. Free boundary problems involving solids, volume 281. CRC Press, 1993.

[5] John M Chadam and Helen Rasmussen. Emerging Applications in Free Boundary Problems, volume 280. CRC Press, 1993.

[6] Luis A Caffarelli, Sandro Salsa, and S Salsa. A geometric approach to free boundary problems, volume 68. American Mathematical Soc., 2005.

[7] Long-Qing Chen. Phase-field method of phase transitions/domain structures in ferroelectric thin films: a review. Journal of the American Ceramic Society, 91(6):1835-1844, 2008.

[8] Ingo Steinbach. Phase-field models in materials science. Modelling and simulation in materials science and engineering, 17(7):073001, 2009.

[9] PG Amos. Understanding the volume-diffusion governed shape-instabilities in metallic systems. arXiv preprint arXiv:1906.10404, 2019.

[10] I Steinbach and F Pezzolla. A generalized field method for multiphase transformations using interface fields. Physica D: Nonlinear Phenomena, 134(4):385-393, 1999.

[11] Britta Nestler, Harald Garcke, and Björn Stinner. Multicomponent alloy solidification: phase-field modeling and simulations. Physical Review E, 71(4):041609, 2005.

[12] Pierre C Hohenberg and Bertrand I Halperin. Theory of dynamic critical phenomena. Reviews of Modern Physics, 49(3):435, 1977.

[13] Samuel M Allen and John W Cahn. A microscopic theory for antiphase boundary motion and its application to antiphase domain coarsening. Acta metallurgica, 27(6):1085-1095, 1979.

[14] Gunduz Caginalp and Paul Fife. Phase-field methods for interfacial boundaries. Physical Review B, 33(11):7792, 1986. 
[15] G Caginalp. Stefan and hele-shaw type models as asymptotic limits of the phase-field equations. Physical Review A, 39(11):5887, 1989.

[16] Adam A Wheeler, William J Boettinger, and Geoffrey B McFadden. Phase-field model for isothermal phase transitions in binary alloys. Physical Review A, 45(10):7424, 1992.

[17] Gunduz Caginalp and Weiqing Xie. Phase-field and sharp-interface alloy models. Physical Review E, 48(3):1897, 1993.

[18] Janin Tiaden, Britta Nestler, Hermann-Josef Diepers, and Ingo Steinbach. The multiphase-field model with an integrated concept for modelling solute diffusion. Physica D: Nonlinear Phenomena, 115(1-2):73-86, 1998.

[19] Seong Gyoon Kim, Won Tae Kim, and Toshio Suzuki. Interfacial compositions of solid and liquid in a phasefield model with finite interface thickness for isothermal solidification in binary alloys. Physical Review E, 58(3):3316, 1998.

[20] Seong Gyoon Kim, Won Tae Kim, and Toshio Suzuki. Phase-field model for binary alloys. Physical review e, 60(6):7186, 1999

[21] Mathis Plapp. Unified derivation of phase-field models for alloy solidification from a grand-potential functional. Physical Review E, 84(3):031601, 2011.

[22] Abhik Choudhury and Britta Nestler. Grand-potential formulation for multicomponent phase transformations combined with thin-interface asymptotics of the double-obstacle potential. Physical Review E, 85(2):021602, 2012.

[23] Larry K Aagesen, Yipeng Gao, Daniel Schwen, and Karim Ahmed. Grand-potential-based phase-field model for multiple phases, grains, and chemical components. Physical Review E, 98(2):023309, 2018.

[24] P-R Cha, D-H Yeon, and J-K Yoon. A phase field model for isothermal solidification of multicomponent alloys. Acta materialia, 49(16):3295-3307, 2001.

[25] Mats Hillert and John Agren. On the definitions of paraequilibrium and orthoequilibrium. Scripta Materialia, 50(5):697-699, 2004.

[26] Dong-Hee Yeon, Pil-Ryung Cha, and Jong-Kyu Yoon. A phase field study for ferrite-austenite transitions under para-equilibrium. Scripta materialia, 45(6):661-668, 2001.

[27] Pil-Ryung Cha, Dong-Hee Yeon, and Jong-Kyu Yoon. Phase-field model for multicomponent alloy solidification. fournal of crystal growth, 274(1-2):281-293, 2005.

[28] Lijun Zhang, Matthias Stratmann, Yong Du, Bo Sundman, and Ingo Steinbach. Incorporating the calphad sublattice approach of ordering into the phase-field model with finite interface dissipation. Acta Materialia, 88:156-169, 2015.

[29] Plapp Mathis. Phase-field modelling of solidification microstructures. Fournal of the Indian Institute of Science, 96(3):179-198, 2016.

[30] PG Kubendran Amos, LT Mushongera, and Britta Nestler. Phase-field analysis of volume-diffusion controlled 
shape-instabilities in metallic systems-i: 2-dimensional plate-like structures. Computational Materials Science, 144:363-373, 2018.

[31] J Eiken, B Böttger, and I Steinbach. Multiphase-field approach for multicomponent alloys with extrapolation scheme for numerical application. Physical review E, 73(6):066122, 2006.

[32] Mats Hillert. Phase equilibria, phase diagrams and phase transformations: their thermodynamic basis. Cambridge University Press, 2007.

[33] PG Kubendran Amos, Ephraim Schoof, Daniel Schneider, and Britta Nestler. Chemo-elastic phase-field simulation of the cooperative growth of mutually-accommodating widmanstätten plates. Fournal of Alloys and Compounds, 767:1141-1154, 2018.

[34] William C Johnson. Precipitate shape evolution under applied stress-thermodynamics and kinetics. Metallurgical and Materials Transactions A, 18(2):233-247, 1987.

[35] PG Kubendran Amos, Avisor Bhattacharya, Britta Nestler, and Kumar Ankit. Mechanisms of pearlite spheroidization: Insights from 3d phase-field simulations. Acta Materialia, 161:400-411, 2018.

[36] PG Kubendran Amos, Ramanathan Perumal, Michael Selzer, and Britta Nestler. Multiphase-field modelling of concurrent grain growth and coarsening in complex multicomponent systems. fournal of Material Science and Technology, Accepted, In-Press.

[37] PG Kubendran Amos, Ephraim Schoof, Daniel Schneider, and Britta Nestler. On the globularization of the shapes associated with alpha-precipitate of two phase titanium alloys: Insights from phase-field simulations. Acta Materialia, 159:51-64, 2018.

[38] Tao Wang, Guang Sheng, Zi-Kui Liu, and Long-Qing Chen. Coarsening kinetics of $\gamma$ precipitates in the ni-almo system. Acta Materialia, 56(19):5544-5551, 2008.

[39] U Grafe, B Böttger, J Tiaden, and SG Fries. Coupling of multicomponent thermodynamic databases to a phase field model: application to solidification and solid state transformations of superalloys. Scripta Materialia, 42(12):1179-1186, 2000.

[40] Qing Chen, Ning Ma, Kaisheng Wu, and Yunzhi Wang. Quantitative phase field modeling of diffusioncontrolled precipitate growth and dissolution in ti-al-v. Scripta Materialia, 50(4):471-476, 2004.

[41] Kaisheng Wu, YA Chang, and Yunzhi Wang. Simulating interdiffusion microstructures in ni-al-cr diffusion couples: a phase field approach coupled with calphad database. Scripta materialia, 50(8):1145-1150, 2004.

[42] Abhik Choudhury, Michael Kellner, and Britta Nestler. A method for coupling the phase-field model based on a grand-potential formalism to thermodynamic databases. Current Opinion in Solid State and Materials Science, 19(5):287-300, 2015.

[43] M Militzer, MG Mecozzi, J Sietsma, and S Van der Zwaag. Three-dimensional phase field modelling of the austenite-to-ferrite transformation. Acta materialia, 54(15):3961-3972, 2006.

[44] PG Kubendran Amos, Ephraim Schoof, Daniel Schneider, and Britta Nestler. On the volume-diffusion governed 
termination-migration assisted globularization in two-phase solid-state systems: insights from phase-field simulations. In Numerical Modelling in Engineering, pages 47-63. Springer, 2018.

[45] Ephraim Schoof, PG Kubendran Amos, Daniel Schneider, and Britta Nestler. Influence of stress-free transformation strain on the autocatalytic growth of bainite: A multiphase-field analysis. Materialia, 9:100620, 2020.

[46] JB Gilmour, GR Purdy, and JS Kirkaldy. Partition of manganese during the proeutectoid ferrite transformation in steel. Metallurgical Transactions, 3(12):3213-3222, 1972.

[47] LT Mushongera, PG Kubendran Amos, B Nestler, and Kumar Ankit. Phase-field simulations of pearlitic divergence in fe-c-mn steels. Acta Materialia, 150:78-87, 2018.

[48] LT Mushongera, PG Kubendran Amos, E Schoof, P Kumar, and B Nestler. The non-steady-state growth of divergent pearlite in fe-c-mn steels: a phase-field investigation. Fournal of Materials Science, 55(12):5280$5295,2020$.

[49] John G Speer, Fernando C Rizzo Assunção, David K Matlock, and David V Edmonds. The" quenching and partitioning" process: background and recent progress. Materials Research, 8(4):417-423, 2005.

[50] J Speer, DK Matlock, BC De Cooman, and JG Schroth. Carbon partitioning into austenite after martensite transformation. Acta materialia, 51(9):2611-2622, 2003.

[51] Y Takahama, MJ Santofimia, MG Mecozzi, L Zhao, and J Sietsma. Phase field simulation of the carbon redistribution during the quenching and partitioning process in a low-carbon steel. Acta materialia, 60(6-7):2916-2926, 2012.

[52] MG Mecozzi, J Eiken, MJ Santofimia, and J Sietsma. Phase field modelling of microstructural evolution during the quenching and partitioning treatment in low-alloy steels. Computational Materials Science, 112:245-256, 2016.

[53] PG Kubendran Amos, Ephraim Schoof, Nick Streichan, Daniel Schneider, and Britta Nestler. Phase-field analysis of quenching and partitioning in a polycrystalline fe-c system under constrained-carbon equilibrium condition. Computational Materials Science, 159:281-296, 2019. 